Illinois State University

ISU ReD: Research and eData

Theses and Dissertations

7-2-2019

\title{
Potential For On-Site, Prosecutorial Evidence From Drug Residues Collected On Plasmonic Paper: A Pilot Study For Sers-Psi-Ms
}

Daniel S. Burr

Illinois State University, daniel.stephen.burr@gmail.com

Follow this and additional works at: https://ir.library.illinoisstate.edu/etd

Part of the Analytical Chemistry Commons, and the Nanoscience and Nanotechnology Commons

\section{Recommended Citation}

Burr, Daniel S., "Potential For On-Site, Prosecutorial Evidence From Drug Residues Collected On Plasmonic Paper: A Pilot Study For Sers-Psi-Ms" (2019). Theses and Dissertations. 1154.

https://ir.library.illinoisstate.edu/etd/1154

This Thesis is brought to you for free and open access by ISU ReD: Research and eData. It has been accepted for inclusion in Theses and Dissertations by an authorized administrator of ISU ReD: Research and eData. For more information, please contact ISUReD@ilstu.edu. 


\section{POTENTIAL FOR ON-SITE, PROSECUTORIAL EVIDENCE FROM DRUG RESIDUES COLLECTED ON PLASMONIC PAPER: A PILOT STUDY FOR SERS-PSI-MS}

\section{DANIEL S. BURR}

67 Pages

Given the potential impact of improvements to on-site drug testing, as well as recent, successful displays of paper spray ionization mass spectrometry (PSI-MS) in this regard, this thesis pilots the implementation of Raman spectroscopy as a compliment to MS for field-based confirmatory drug testing. Surface enhanced Raman scattering (SERS) is utilized for applications to trace detection. Two-tiered analysis of individual drug samples is enabled using triangularlycut plasmonic papers, from which both SERS and PSI-MS analysis may be performed. Several drug compounds, representative of traditional and emerging drug types, are examined by these techniques, both separately and as a fully integrated, SERS-PSI-MS testing platform. Calibration models for the drugs are constructed, which indicate single-nanogram limits of detection. The unique benefits offered by Raman/SERS are demonstrated via discrimination of isomers that are indistinguishable by MS. Finally, optimization of the coupled, analytical platform culminates in a standard operating procedure. This is implemented in an error-rate study $(N=500)$ performed for sub-microgram quantities of drugs, returning a $99.2 \%$ success rate.

KEYWORDS: Raman spectroscopy; surface enhanced Raman scattering (SERS); ambient mass spectrometry; portable forensics; controlled substances; plasmonics 
POTENTIAL FOR ON-SITE, PROSECUTORIAL EVIDENCE FROM DRUG RESIDUES

COLLECTED ON PLASMONIC PAPER: A PILOT STUDY FOR SERS-PSI-MS

DANIEL S. BURR

A Thesis Submitted in Partial Fulfillment of the Requirements for the Degree of

MASTER OF SCIENCE

Department of Chemistry

ILLINOIS STATE UNIVERSITY

2019 
(C) 2019 Daniel S. Burr 
POTENTIAL FOR ON-SITE, PROSECUTORIAL EVIDENCE FROM DRUG RESIDUES COLLECTED ON PLASMONIC PAPER: A PILOT STUDY FOR SERS-PSI-MS

DANIEL S. BURR

COMMITTEE MEMBERS:

Jeremy Driskell, Chair

Jun-Hyun Kim

Michael Webb 


\section{ACKNOWLEDGMENTS}

There are many people I wish to thank for making this work possible. First, the one for whom this degree cost the most - my bride and my love, Kayla. Through many exhausting days and weeks with our children, still you have been an inexhaustible encouragement to me. For each smile, each meal, and each extra hour you've consented to my absence, thank you. We finally made it! Second, I thank my advisor, Dr. Jeremy Driskell. You have been a model to me - not only as a chemist - but as a dedicated worker. I thank Dr. Jun-Hyun Kim and Dr. Michael Webb for serving on my thesis committee, reading and carefully considering my work. Thanks goes to Dr. Christopher Mulligan - the principle investigator for the collaborative project of which this work was a part. Through his efforts, together with Dr. Jamie Weiland, and, again, Dr. Driskell and Dr. Kim, I was privileged to work on a very intriguing research project. I thank Dr. Jean Standard, who graciously taught and assisted with computational methods. Many thanks go to my colleagues on this project: William Fatigante, John Harms, Jemima Lartey, Ashley Stelmack, and Noah McLurg. I thank my associates in the Driskell and Kim research groups, who added character and relish to my time as a research assistant: Kiran Tripathi, Richard Frimpong, Wongi Jang, Pascal Eyimegwu, Samuel Okyem, Olatunde Awotunde, Guadalupe Ruiz, and Evan Strandquist. I also thank those in the Department of Chemistry whose guidance and company I enjoyed - particularly Tony Ludwig, Megan Tolle, Ginger Beer, Shanna McDaniels, and Dr. Craig McLauchlan. I thank Illinois State University as a whole - my time as a student was very blessed and will be fondly remembered for many years. Finally and most completely, I give thanks to the Lord for all He has brought me through, the family He has blessed me with, and the work He has given me - both accomplished and yet to come.

D. S. B. 


\section{CONTENTS}

Page

ACKNOWLEDGMENTS

CONTENTS

TABLES

FIGURES

CHAPTER I: INTRODUCTION 1

Scope of Forensic Drug Evidence Problem 1

Forensic Drug Testing Guidelines $\quad 2$

On-site Detection Strategies $\quad 3$

Colorimetric Testing on Paper Analytical Devices 3

Electrochemical Detection $\quad 4$

Portable, Ambient Ionization Mass Spectrometry 5

Portable Raman Spectroscopy $\quad 7$

Surface-enhanced Raman Scattering (SERS) $\quad 8$

$\begin{array}{lr}\text { SERS Substrates } & 8\end{array}$

$\begin{array}{ll}\text { Paper SERS Substrates } & 10\end{array}$

SERS for the Detection of Controlled Substances $\quad 12$

$\begin{array}{ll}\text { SERS-PSI-MS } & 13\end{array}$

CHAPTER II: EXPERIMENTAL METHODS 15

$\begin{array}{ll}\text { Reagents } & 15\end{array}$

$\begin{array}{ll}\text { Preparation of Plasmonic Papers } & 15\end{array}$

$\begin{array}{ll}\text { Instrumental Methods } & 16\end{array}$ 
$\begin{array}{ll}\text { Raman Spectroscopy } & 16\end{array}$

$\begin{array}{ll}\text { Paper Spray Ionization Mass Spectrometry } & 18\end{array}$

$\begin{array}{ll}\text { Integrated SERS-PSI-MS } & 20\end{array}$

$\begin{array}{ll}\text { Method development and staging } & 20\end{array}$

$\begin{array}{ll}\text { Standard operating procedure } & 21\end{array}$

$\begin{array}{ll}\text { SERS Identification of Drugs } & 21\end{array}$

SERS Limits of Detection $\quad 22$

Density Functional Theory: Calculated Spectra 23

$\begin{array}{ll}\text { Discrimination among Isomers Using SERS } & 23\end{array}$

$\begin{array}{ll}\text { SERS-PSI-MS Error-rate Study } & 24\end{array}$

Method Validation $\quad 24$

$\begin{array}{ll}\text { Error-rate Study } & 25\end{array}$

CHAPTER III: RESULTS \& DISCUSSION 26

SERS Detection of Drugs 26

Optimizing SERS Sensing on Plasmonic Papers 26

SERS of Representative Drugs $\quad 29$

Spot-to-spot and Batch-to-batch Reproducibility 31

Limits of Detection 33

Conformity to Calculated Spectra $\quad 44$

SERS Discrimination of Isomers 46

$\begin{array}{ll}\text { SERS-PSI-MS } & 51\end{array}$

$\begin{array}{ll}\text { Error-rate Study } & 55\end{array}$

CHAPTER IV: CONCLUSIONS AND OUTLOOK 57 
REFERENCES 


\section{TABLES}

Table

Page

1. SWGDRUG categorization of analytical techniques for drug analysis

2. Performance of recently reported plasmonic paper substrates

3. Reproducibility of SERS response on plasmonic paper by drug

4. Limits of detection for five representative drugs on plasmonic paper

5. Intra-day and inter-day variabilities in SERS drug identification performance

for coupled SERS-PSI-MS analysis 


\section{FIGURES}

Figure

Page

1. Representation of the simplistic operation of PSI-MS 6

2. Scheme for combined approach to trace drug detection by SERS-PSI-MS 14

3. Process of preparing plasmonic papers 16

4. TSI ProRaman-L with attachments 17

5. FLIR Systems Griffin AI-MS 1.2, with attached Thorlabs optomechanical cage system

6. Precision sample stage, supplemented with 3D-printed devices, for integrated SERS-PSI-MS analysis

7. Representative drug compounds used in this study 22

8. Structural and positional isomers selected to assess SERS discriminating 24 power

9. Optimization of power settings for the $785 \mathrm{~nm}$ Raman excitation laser on dry plasmonic paper

10. Image capturing the lack of homogeneity in AuNP loading on plasmonic paper

11. Raman acquisitions collected from a punch of plasmonic paper, loaded with 1 $\mu \mathrm{g}$ fentanyl

12. Normalized, offset Raman spectra of $1 \mu \mathrm{g}$ each of $2 \mathrm{C}-\mathrm{B}$, cocaine, fentanyl, hydrocodone, and JWH-018 on unmodified and plasmonic paper

13. Overlaid SERS spectra for 333 ng of fentanyl, collected from different locations on a single plasmonic substrate and three independently prepared substrates 
14. Overlaid SERS spectra and calibration curve for 2C-B 36

15. Overlaid SERS spectra and calibration curve for cocaine 37

16. Overlaid SERS spectra and calibration curve for fentanyl 38

17. Overlaid SERS spectra and calibration curve for hydrocodone 39

18. Overlaid SERS spectra and calibration curve for JWH-018 40

19. Truncated, overlaid spectra of plasmonic paper with no analyte, $5 \mathrm{ng} 2 \mathrm{C}-\mathrm{B}$,

and 83 ng 2C-B $\quad 42$

20. Normalized SERS response of plasmonic paper before and after the deposition

of trisodium citrate $\quad 43$

21. DFT-calculated spectra overlaid with SERS spectra for each drug compound 45

22. The tryptamine derivatives psilocin and bufotenine 47

23. Isomers of JWH-018 50

24. Representative data for SERS-PSI-MS, collected from $100 \mathrm{ng}$ of $2 \mathrm{C}-\mathrm{B}$ on

plasmonic paper $\quad 52$

25. Plasmonic, PSI-cut paper with SERS analysis conducted before and after PSI-

MS for all five drugs $\quad 54$ 


\section{CHAPTER I: INTRODUCTION}

\section{Scope of Forensic Drug Evidence Problem}

Data collected by the Bureau of Justice Statistics have shown that, in publicly funded forensics laboratories, substantial time and effort are exhausted on the routine analysis of suspected controlled substances. ${ }^{1}$ In 2014 specifically, drug testing commanded a steady one-third of all forensics case requests; such testing was similarly represented within the 570,000 national sample backlog. Increasing sample throughput could expedite confirmatory drug testing, forensics testing generally, and legal proceedings. Overall, significant public resources could be conserved by streamlining the handling of drug evidence.

In current practice, drug testing follows a deliberate, nearly universal protocol. ${ }^{2}$ Evidence is first encountered by police officers, narcotics agents, or detectives operating in various locales (e.g. traffic stops, clandestine labs, drug house busts). Once seized, samples are presumptively identified via a colorimetric field-test. After this they are transported to forensics laboratories for confirmatory analysis, which is performed using established instrumental techniques - most often gas chromatography coupled with mass spectrometry (GC-MS).

Though it is relatively straightforward, this process leaves much room for improvement. The time required for each sample is extensive, spanning seizure, presumptive identification, transport, time in a work queue, and analysis. Current presumptive tests have multiple drawbacks, ${ }^{3,4}$ which allow false positives to be carried through the process until its end, adding unnecessary work for law enforcement and forensics practitioners. The situation is further complicated by emerging classes of drugs, e.g. new psychoactive substances (NPSs) and synthetic opioids. ${ }^{5}$ There is widespread frenzy regarding how to deal with such substances, which elude 
detection by continuously appearing in derivatized forms, and - owing to their dangerously high potency - are often present at low concentrations. ${ }^{5}$

Ultimately, forensics laboratories face a burden they are not equipped to carry. Revisiting forensics best-practices, along with considering the potential of alternate detection strategies, is requisite for redirecting this strain from lab to field. The modernization of field-testing - arguably the best focus for streamlining drug analysis - is an active, widespread research effort. ${ }^{6}$

\section{Forensic Drug Testing Guidelines}

To hold merit in the forensics community, testing strategies must align with established recommendations, such as those of the Scientific Working Group for the Analysis of Seized Drugs (SWGDRUG). To establish minimum requirements for accurate, court-admissible drug evidence, SWGDRUG ranks analytical techniques according to their discriminating power (Table 1). Drug evidence is deemed admissible only after positive identification by two independent techniques, as long as one technique resides in Category A. ${ }^{7}$ Conforming to SWGDRUG recommendations would be an important benchmark in the development of a novel, on-site drug testing strategy.

Table 1

SWGDRUG categorization of analytical techniques for drug analysis

\begin{tabular}{ccc}
\hline Category A & Category B & Category C \\
\hline Infrared spectroscopy (IR) & Capillary electrophoresis (CE) & Color tests \\
Mass spectrometry (MS) & Gas chromatography (GC) & Fluorescence \\
Nuclear magnetic resonance & Ion mobility spectrometry (IMS) & Immunoassay \\
Raman spectroscopy & Liquid chromatography (LC) & Melting point \\
X-ray diffractometry & Thin layer chromatography (TLC) & UV-vis \\
& & spectrophotometry \\
\hline
\end{tabular}


Many Category A and B techniques are commercially available in portable formats (e.g. IR, Raman, MS). Implementation of one such method as an improved presumptive test would reduce the workload caused by false positives and ambiguous results. However, keeping in mind SWGDRUG's two-tiered recommendation, the combined use of two independent portable techniques could generate admissible evidence entirely in the field.

\section{On-site Detection Strategies}

The ability to rapidly, reliably identify samples at the point they are first encountered would constitute a desirable paradigm shift in the handling of drug evidence. Currently, field-based testing is limited to colorimetric reagent test kits. Under this methodology, a suspicious sample is mixed with a reagent cocktail (often Marquis or Scott) that characteristically changes color in the presence of common drugs of abuse. Such tests are rapid and easy to use, yet they have many drawbacks, including false positives, ${ }^{3}$ ambiguous results, ${ }^{4}$ a lack of chemical specificity, ${ }^{8}$ and applicability to only a handful of anticipated analytes. Foregoing colorimetric detection, there is a repertoire of portable, analytical methods that are inherently more selective and broadly applicable. It is beyond the scope of this work to review all of them, however, and so focus will be directed principally towards the Category A techniques of vibrational spectroscopy and portable MS. An emerging area of portable, trace drug detection, e.g. electrochemical methods - though not yet ranked by SWGDRUG - is also discussed.

\section{Colorimetric Testing on Paper Analytical Devices}

Several methods for overcoming these deficits have been reported. Improved colorimetric testing has been demonstrated on paper analytical devices (PADs). Notably, one PAD used waxprinted, branched reservoirs of reagents to multiplex color testing. ${ }^{9}$ This design proffered additional analytical information while purportedly retaining ease of use. However, the 
interpretation of such tests quickly becomes complex, with multiple color changes across several reaction wells. To overcome this difficulty, and improve sensitivity, detection in some of these systems has been extended beyond visualization - relying instead on computerized detection of fluorescence or luminescence. ${ }^{10,11}$ Yet, in these cases, simplistic design and operation are traded for only modest increases in discriminatory power. If computerized detection is to supersede visual interpretation, a colorimetric approach loses its fundamental appeal.

\section{Electrochemical Detection}

The principle behind electrochemical detection is that drug molecules can be identified from their oxidation/reduction potentials, which are adequately specific for each molecule. Electrochemical methods are an emerging trace detection strategy for forensics, ${ }^{12}$ and have recently been used for the detection of cocaine (along with its common adulterants), ${ }^{13}$ new psychoactive substances, ${ }^{14}$ and synthetic cannabinoids. ${ }^{15}$ These methods boast a range of benefits, including low cost, simplistic design, and commercially available, portable, battery-powered instrumentation.

Traditionally, electrochemical systems suffer from electrode fouling, limited throughput, and poor selectivity. Recent work has circumvented these problems in a variety of ways. Electrode fouling has been mitigated through the use of screen-printed electrodes (SPEs), which simplify the handling of electrodes and are intended for single or limited use. Additionally, sample throughput has been improved using batch-injection analysis (BIA) systems. ${ }^{16}$ Within these systems, small samples $(\sim 50 \mu \mathrm{L})$ are injected onto the working electrode in an electrochemical cell within which a bulk liquid is stirred or flowed. Oxidation/reduction is briefly detected, and while the electrode is stripped the sample is diluted into the larger volume - readying the cell for the next sample. Coupling of BIA with SPEs has been used to achieve throughputs of 60 sample injections per 
hour. ${ }^{17}$ Improvements to selectivity have involved immobilized molecules - enzymes, ${ }^{18}$ antibodies, ${ }^{19}$ aptamers ${ }^{20}$ and molecular imprinted polymers ${ }^{21}$ - as well as electrodes on which drug analytes have an affinity or a particular tendency to be electroactive. ${ }^{22}$ However, neither of these improvements to selectivity can be simultaneously optimized for many classes of drug molecules. Depending on the electrode, some drug molecules may not be electroactive at all. ${ }^{23}$ Overall, recent advances - along with inherently simple instrumentation - have made portable electrochemical detection a feasible, promising option. However, electrochemical activity remains a modestly selective parameter. Furthermore, the best developments in throughput and ease of operation (BIA-SPE) still require the preparation and pipetting of dissolved samples - these could be considered detriments toward the goal of field-based use by non-chemists.

\section{Portable, Ambient Ionization Mass Spectrometry}

Among portable instrumental techniques for field-based drug analysis, ambient ionization mass spectrometry (AI-MS) has arguably the most promise, especially for its sensitivity, selectivity (particularly with tandem MS capabilities), and minimal requirements for sample manipulation. ${ }^{24-27}$ Samples are ionized under ambient conditions and transferred through a vacuum gradient into the instrument for mass analysis and detection. A variety of ambient ionization methods have been reported during the last two decades, including desorption electrospray ionization (DESI) ${ }^{28,29}$ direct analysis in real time (DART), ${ }^{30,31}$ low temperature plasma (LTP), ${ }^{32,33}$ and - more recently - paper spray ionization (PSI). ${ }^{34,35}$

In PSI-MS (Figure 1), sample is deposited onto a piece of triangularly-cut paper (typically Whatman, blotting, or another. The paper is then positioned in front of an AI-MS, with an applied potential (usually several $\mathrm{kV}$ ) between the paper and the inlet capillary. With the addition of solvent, sample is wicked through the pores until it reaches the tip, whereupon an electrospray is 
formed. Akin to traditional electrospray ionization, it is within this spray that gas-phase analyte ions are formed and guided into the mass spectrometer.

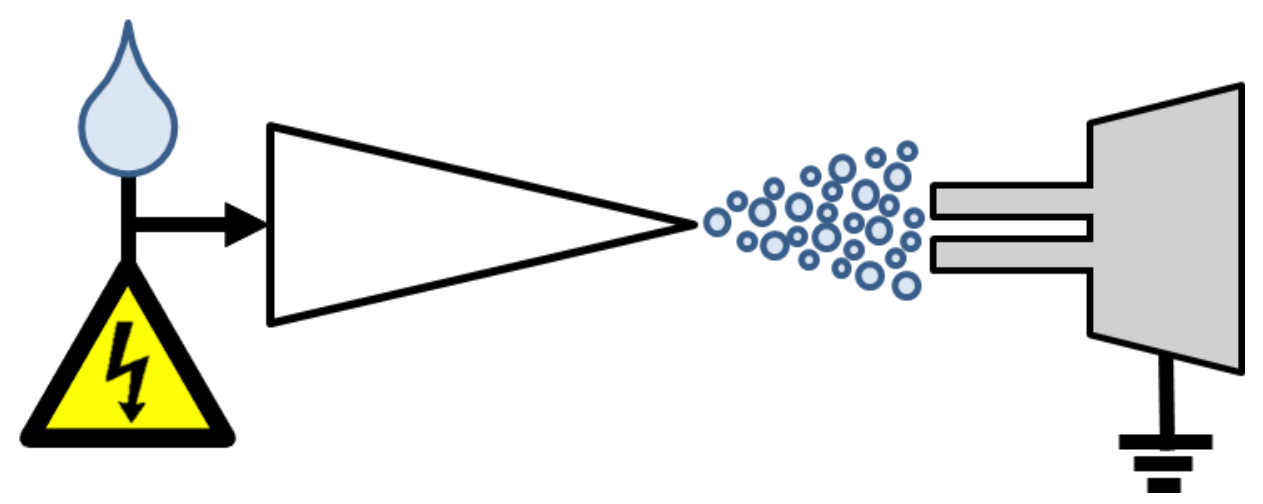

Figure 1. Representation of the simplistic operation of PSI-MS. A triangularly-cut paper, containing the sample, is subjected to high voltage and then wetted with solvent. This results in the generation of an electrospray at the paper tip. Gas phase analyte ions are formed from this spray and, with minimal positioning requirements, enter the mass spectrometer.

PSI-MS holds some distinct advantages over other AI-MS methods. Firstly, the paperbased ionization source can be used as a swab for collecting samples. This, along with its simplistic execution, makes PSI-MS very amenable to use by minimally trained personnel. Since PSI incorporates paper chromatography, it is possible to separate analyte compounds from complex matrices, as has been demonstrated for dried blood spots and foodstuffs. ${ }^{34,36}$ Furthermore, application of PSI to drug analysis on a portable, AI-MS systems has been the subject of extensive work. ${ }^{37-40}$

While portable AI-MS is able to meet many of the demands specific to on-site drug detection, the instrumentation is expensive, and this could be considered prohibitive. However, a recent statistical model explored the benefits (financial and otherwise) to be anticipated from 
routine use of such a platform. ${ }^{41}$ While the initial cost is high, the reduced number of false positives forecast a net reduction in cost. There is thus a strong case that AI-MS is not only analytically powerful and amenable to field-based drug detection - but that it is also financially viable.

\section{Portable Raman Spectroscopy}

Vibrational spectroscopy (IR and Raman) is extremely selective in that it detects the energies of molecular vibrations. Every molecule's various functional groups and connectivity give rise to unique, quantized vibrational energies. Taken together, these energy transitions provide a molecular fingerprint, which is very useful for chemical identification purposes. For complex mixtures, where the fingerprints of many compounds overlap, multivariate statistical tools spectra can be used to deconvolute spectra, allowing the identification of its constituents. ${ }^{42}$ Vibrational spectra also display good selectivity in the cases of isomeric compounds ${ }^{43,44}-$ a challenge for other highly-selective portable detection strategies (e.g. MS).

Both IR and Raman have long met with success in forensics applications. ${ }^{45}$ Compared with IR, however, Raman proffers minimal interference from water, sharp peaks with high signal-tonoise ratio, and instrumentation that tends to be simpler and more affordable (ranging from $\sim 5,000-\$ 50,000)$. Portable Raman spectrometers have been demonstrated in the detection of various pathogens, ${ }^{46-50}$ counterfeit pharmaceuticals, ${ }^{51-53}$ and controlled substances.${ }^{54-56}$

One drawback of vibrational spectroscopy is its limited applicability to trace evidence - IR and Raman are better suited for bulk analysis. AI-MS methods, on the other hand, are sensitive to nanogram quantities of material. A unique benefit of Raman, however, is that it can be extended to trace detection through the phenomenon of surface-enhanced Raman scattering (SERS). 


\section{Surface-enhanced Raman Scattering (SERS)}

SERS, initially discovered in $1977,,^{57,58}$ is a phenomenon wherein molecules in close proximity (single nanometers) to noble-metal nanostructures elicit highly intensified Raman scattering. Reported enhancement factors (EFs) have ranged from $10^{4}$ to $10^{14}$, and SERS applications have been reported to reach single molecule sensitivities. ${ }^{59,60}$ The enabling medium for this ultrasensitive detection is a suitable SERS - often termed plasmonic - noble metal substrate.

The conduction band electrons of metal nanostructures tend to behave as collectives called plasmons - which can oscillate in frequency with electromagnetic radiation. The specific wavelength at which plasmons experience maximal oscillations is termed the surface plasmon resonance (SPR). For noble metals this occurs at visible frequencies. Upon irradiation with visible light, then, the immediate surroundings of noble metal nanostructures experience highly enhanced electric fields. Molecules adsorbed onto the surface of these structures, owing to the enhanced electric fields, scatter Raman photons several orders of magnitude more intensely than under traditional, bulk conditions. ${ }^{61}$

\section{SERS Substrates}

Since the first investigations into SERS there has been a steady lineage of plasmonic - and thus surface-enhancing - substrates. This progression is delineated in many SERS reviews, past and present. ${ }^{62-65}$ Prerequisite to reviewing the varieties of plasmonic substrates, however, there are several common metrics to note.

The first of these is the previously mentioned EF, which assesses the degree to which Raman scattering is increased. Finding a single, adequate definition of EFs has been a challenge within the SERS community, and many ways of calculating EFs exist and are reported. ${ }^{61}$ In part 
this is because of the many factors involved in SERS, namely the interactions between analyte and plasmonic surfaces. Maximal SERS enhancement is observed when molecules are within $1 \mathrm{~nm}$ of the plasmonic surface. Furthermore, specific vibrations closer and more orthogonal to the surface experience the greatest enhancement. Since different molecules will have different affinities for the surface and different preferred orientations, the EF is not a one-size-fits-all metric.

Beyond the EF, important assessments of a substrate are the consistency of its performance from one spot to another (spot-to-spot variation), its reproducibility (batch-to-batch variation), and its performance with time (stability or day-to-day variation). These are usually reported as percent relative standard deviations (\%RSD) for the intensity of a single peak. For spot-to-spot and batchto-batch variations, values of $10 \%$ and lower are usually regarded as excellent. Stability is ideally on the order of weeks or months, but due to fouling of gold or (especially) silver nanostructures, the consistent performance of substrates across days or weeks is considered satisfactory.

SERS was a serendipitous discovery by two independent research groups, who both happened upon anomalously intense signals when using Raman spectroscopy to monitor chemical activity on electrochemically-roughened silver electrodes. ${ }^{57,58}$ Due to the simple preparation and adequate EFs of electrochemically-roughened films, these became common early SERS substrates. However, batch-to-batch reproducibility is a severe limitation: substrates prepared under identical conditions can vary in signal intensities by $40 \% .^{66}$

Colloidal suspensions of gold or silver nanoparticles (AuNPs, AgNPs) are very common SERS substrates. Nanoparticles are synthesized by aqueous reduction of gold or silver salts, stabilized with a capping agent (usually the same species used in reduction, e.g. citrate), and aggregated with electrolytes such that the SPR is shifted to the ideal location. This tunability is both a strength and a weakness for colloidal substrates. The size, geometry, and extent of 
aggregation can all be adjusted to provide strong EFs. However, reproducibility is poor: factors such as timing and mixing can cause polydispersity in the nanoparticles - and aggregation is a random process, which is time-dependent and non-specifically triggered. ${ }^{62}$ Additions of sample to the delicately-balanced colloids, if they affect the solution as a whole, can cause nanoparticles to massively aggregate and crash from solution. A way to circumvent this is to immobilize the nanoparticles on a solid support - even then, however, the aggregation process is difficult to control, and reproducibility can be an issue.

In order to circumvent problems with reproducibility, many solid-phase nanofabrication strategies have been used for SERS substrate design. Vapor deposition methods, electron-beam etching, and nanolithography techniques are among the tools for producing solid-phase plasmonic substrates with maximal enhancement factors and reproducibility. ${ }^{64}$ Such rigorous, tightly controlled synthetic methods were (and are) vital to the advancement of SERS theory. However, with the time, equipment, and expertise required to prepare such substrates, there has arisen a separate body of research, directed towards low-cost, high-throughput preparation of plasmonic substrates. Insofar as such substrates have demonstrated adequate enhancement and reproducibility, SERS has become an increasingly field-ready technique.

\section{Paper SERS Substrates}

Plasmonic paper substrates are at the forefront of low-cost, high-throughput options for SERS sensing. ${ }^{63,67}$ Paper is a three-dimensional, porous framework of cellulose fibers. As a result of this porosity, samples spontaneously wick through it. This makes paper SERS sensing versatile:

reports have leveraged the porous nature of paper for ease of sampling (as dipsticks and swabs), ${ }^{68}$ as well as for paper chromatography and lateral flow preconcentration. ${ }^{69,70}$ 
Paper has been rendered plasmonic via treatment with Au or Ag colloids, either from soaking, ${ }^{71,72}$ spraying, ${ }^{73}$ or printing. ${ }^{68-70,74}$ In the former, colloids are directly applied onto paper. In the latter two, colloids are rendered more viscous - usually with added glycerol - to form a stable, nanoparticle ink. Self-assembled films, formed at the interface of colloidal nanoparticles with organic solvents, have also been deposited onto filter papers. ${ }^{75}$ Alternatively, papers have been endowed with plasmonic structures in situ, by electroless deposition (e.g., silver-mirror reaction $)^{76}$ as well as photo-assisted citrate reduction of $\mathrm{AgNO}_{3}{ }^{77}$

The challenge for paper SERS substrates has been to demonstrate good enhancement and reproducibility. Table 2 summarizes some of the metrics achieved by modern plasmonic papers, all of which are satisfactory. Stability is not reported, nor is throughput - these are among the important factors for distinction among substrates, all else being equal. 
Table 2

Performance of recently reported plasmonic paper substrates: enhancement factors (EFs), limits of detection (LODs), percent relative standard deviations (\%RSD), and model analytes

\begin{tabular}{|c|c|c|c|c|c|c|}
\hline Method & Nanoparticle & $\mathbf{E F}$ & LOD & \%RSD & Analyte & Ref. \\
\hline Dripping & $\begin{array}{c}\text { AuNS } \\
\text { (nanostar) }\end{array}$ & $1.2 \times 10^{7}$ & $1 \mathrm{nM}$ & NR & Crystal violet & [78] \\
\hline Soaking & $\begin{array}{c}\text { AuNR } \\
\text { (nanorod) }\end{array}$ & $5 \times 10^{6}$ & $\begin{array}{c}140 \mathrm{pg} \\
\text { (swabbing) }\end{array}$ & NR & 1,4-benzenedithiol & [79] \\
\hline Spraying & $\mathrm{AgNP}$ & $2 \times 10^{7}$ & NR & $\begin{array}{l}14.5 \% \\
\text { (spot) }\end{array}$ & Rhodamine $6 \mathrm{G}$ & [73] \\
\hline Printing & AgNP & $2 \times 10^{5}$ & $4 \mathrm{pg}$ & $\begin{array}{l}15 \% \\
\text { (spot) }\end{array}$ & $\begin{array}{c}\text { EF: Rhodamine } 6 \mathrm{G} \\
\text { LOD: } 1,2-\mathrm{Bis}(4- \\
\text { pyridyl)ethylene }\end{array}$ & $\begin{array}{l}{[68]} \\
{[74]}\end{array}$ \\
\hline $\begin{array}{l}\text { Self- } \\
\text { assembly }\end{array}$ & $\mathrm{AgNP}$ & NR & $50 \mathrm{nM}$ & $\begin{array}{c}<5 \% \\
\text { (batch) }\end{array}$ & Rhodamine B & {$[75]$} \\
\hline Electroless & $\mathrm{AgNP}$ & $1.9 \times 10^{7}$ & $625 \mathrm{nM}$ & $\begin{array}{c}8 \% \\
\text { (batch) }\end{array}$ & $\begin{array}{l}\text { EF: 4-nitrobenzenethiol } \\
\text { LOD: tyrosine }\end{array}$ & [76] \\
\hline Electroless & AuNP & $7.8 \times 10^{8}$ & $1 \mathrm{pM}$ & $\begin{array}{c}7.5 \% \\
\text { (batch) }\end{array}$ & 2-naphthalenethiol & [80] \\
\hline $\begin{array}{l}\text { Photo- } \\
\text { assisted } \\
\text { deposition }\end{array}$ & $\mathrm{AgNP}$ & $2.1 \times 10^{7}$ & $160 \mathrm{nM}$ & $\begin{array}{c}8 \% \\
(\text { spot })\end{array}$ & Adenine & [77] \\
\hline
\end{tabular}

\section{SERS for the Detection of Controlled Substances}

There are several reviews describing applications of SERS in drug testing, as well as for forensic science generally, utilizing many of the aforementioned substrates ${ }^{81,82}$ Colloidal AuNPs and AgNPs have been used to detect analytes such as synthetic cannabinoids, ${ }^{83}$ abused pharmaceuticals, ${ }^{84}$ and well known street drugs such as cocaine and MDMA ${ }^{85}$ There are fewer reports of solid-phase SERS substrates (barring paper) used for illegal drugs, but the list of analytes 
is still extensive, including benzodiazepines,${ }^{86}$ cocaine, ${ }^{87}$ and - specifically in the case of AuNPendowed fused-silica capillaries - broad swaths of common narcotics. ${ }^{88,89}$ Reports on paper SERS substrates, perhaps since they are geared towards field-based applications, very often include one or several illegal drug analytes.

\section{SERS-PSI-MS}

A satisfactory, field-based forensics testing platform quickly delivers accurate information while remaining easy, safe, and reliable for non-technical personnel to use under the constraints of law enforcement settings (e.g. limited time, scant evidence, potentially threatening circumstances). An ideal system - rising to SWGRUG guidelines - incorporates two independent techniques, such that evidence can be both screened and confirmed in the field. Generating field-based, courtadmissible evidence would streamline drug analysis, saving significant public resources.

Among the many options for portable detection, Raman/SERS and MS are unparalleled in sensitivity, selectivity, rapidity, and ease of use. There is overlap between a particularly field-ready AI-MS technique - PSI-MS - and a maturing SERS technique - the use of paper substrates. Using plasmonic paper samples could allow for efficient coupling of these two powerful detection strategies (Figure 2). The remainder of this work reports proof-of-principle efforts to combine SERS and PSI-MS, performing both techniques from a single plasmonic paper. Prerequisite to this coupled analysis, SERS is established as a promising compliment to MS in terms of sensitivity (LODs) and selectivity (discrimination among isomers). 


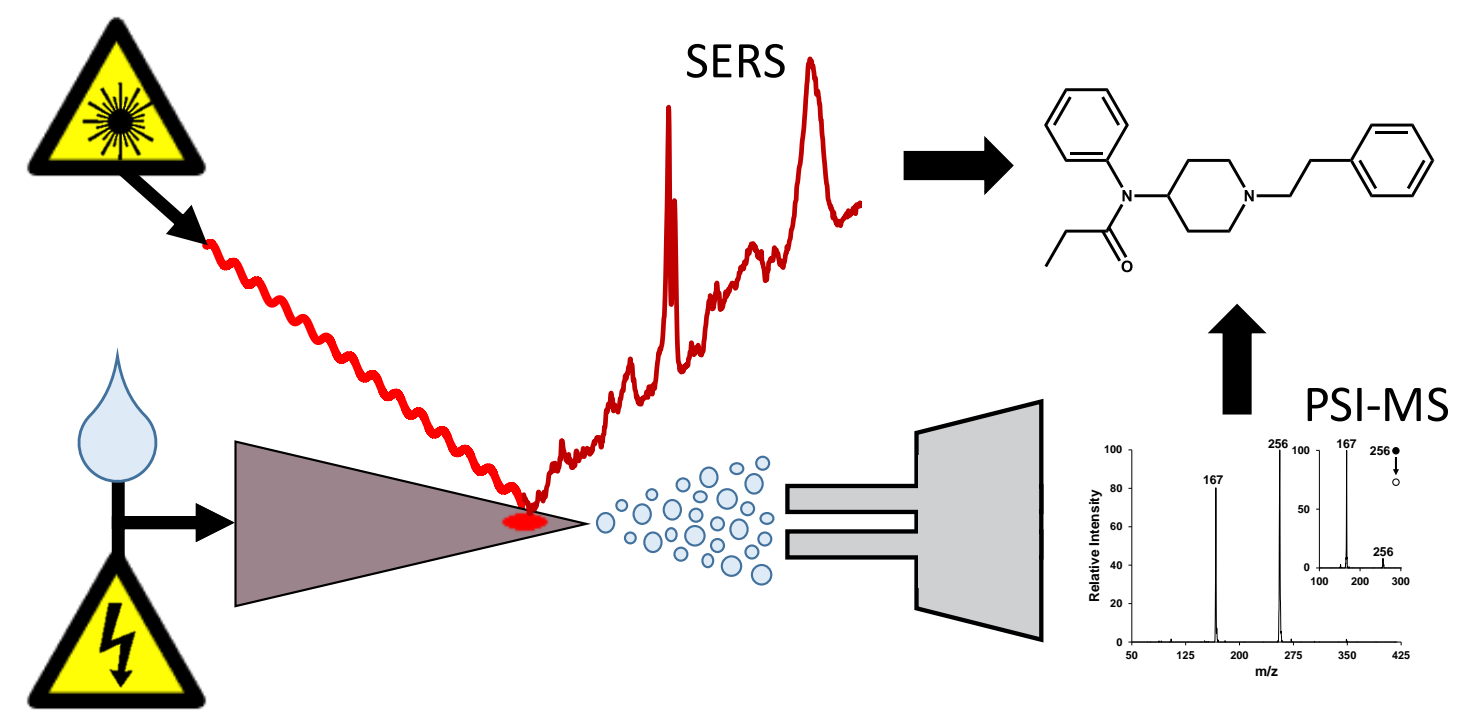

Figure 2. Scheme for the combined approach to trace drug detection by SERS-PSI-MS. The enabling medium is a sharp-tipped plasmonic paper, from which both PSI-MS and SERS may be performed - on a single deposited sample. 


\section{CHAPTER II: EXPERIMENTAL METHODS}

\section{Reagents}

Tetrachloroauric acid trihydrate (ACS regent grade) and trisodium citrate dihydrate (99\%) were obtained from Acros Organics (New Jersey, USA). Whatman Grade 1 filter paper

(GE Healthcare) was used for all plasmonic paper substrates. 2C-B, cocaine, fentanyl, and hydrocodone standard solutions were purchased from Cerilliant (Texas, USA) as $1 \mathrm{mg}$ dissolved in $1 \mathrm{~mL}$ of methanol. Psilocin and bufotenine $(1 \mathrm{mg})$ and JWH-018 $(5 \mathrm{mg})$ were purchased from Cayman Chemical Company (Michigan, USA). All drug samples were DEA-exempt. HPLCgrade methanol and formic acid (99.5\%) were acquired from Fisher Scientific (New Jersey, USA). $18 \mathrm{M} \Omega$ water was used for all solvents.

\section{Preparation of Plasmonic Papers}

Gold nanoparticles (AuNPs) were prepared by thermal reduction of aqueous tetrachloroauric acid with citrate. Resulting AuNPs were characterized using an Agilent 8453 $\mathrm{UV}$-vis spectrophotometer to screen for the desired surface plasmon resonance (SPR) peak, in this case within $535-540 \mathrm{~nm}$. Colloids that met this specification were evenly pipetted over Whatman Grade 1 filter papers - contained in Petri dishes - until each paper was submerged. Following this, the papers were left to soak for 24 hours in room air, with the petri dishes partially lidded to avert dust. Papers - now colored purple - were removed and left in room air to dry (Figure 3). Residual colloids were reevaluated by UV-vis to assess concentration of AuNPs remaining in suspension. This was used as a rough metric to describe the extent of AuNP loading on the paper. AuNPs adsorbed onto the paper were also visualized by electron microscopy, using a FEI Quanta 450 environmental SEM. 


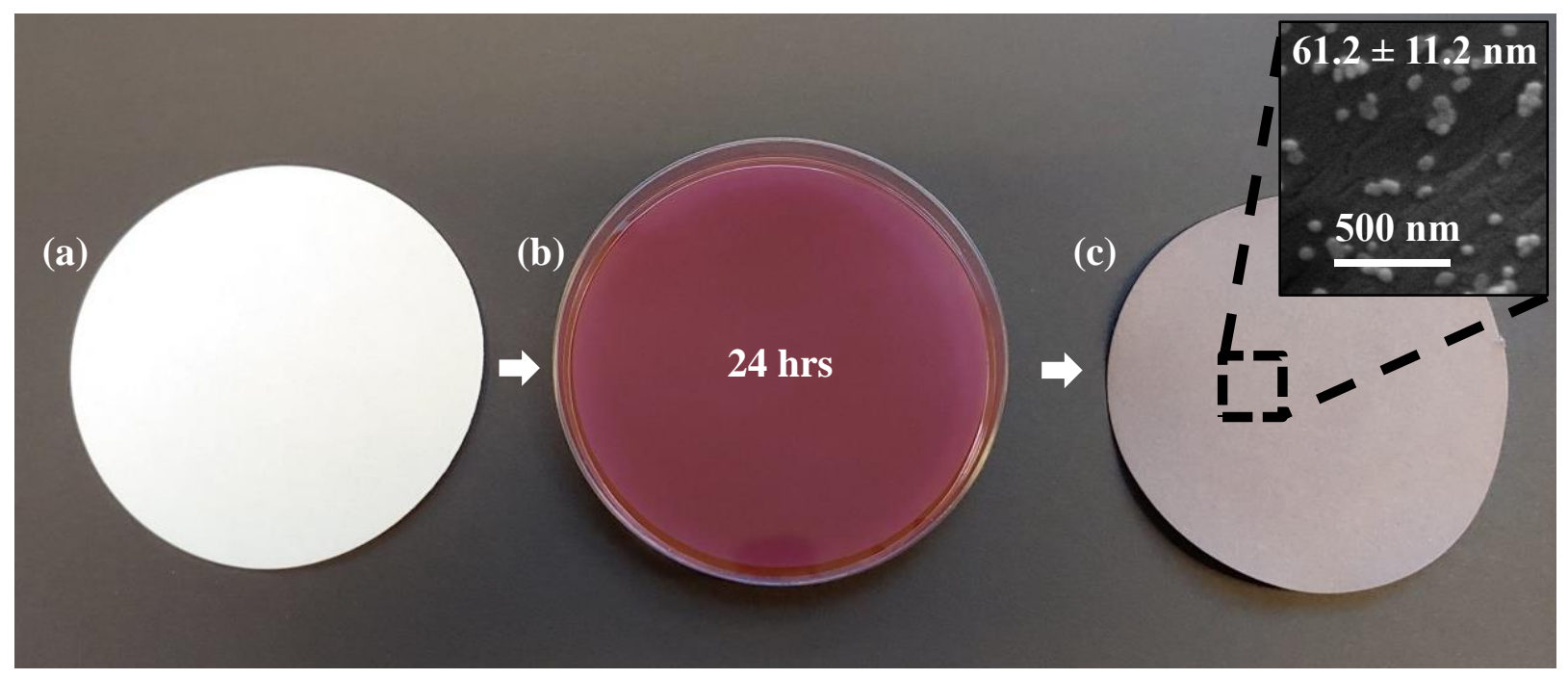

Figure 3. Process of preparing plasmonic papers. Whatman Grade 1 filter papers (a) are immersed in colloidal AuNP (b), then removed after 24 hours to dry (c). The inlaid SEM micrograph shows a single cellulose fiber within a filter paper, onto which AuNPs have adsorbed and formed small aggregates.

\section{Instrumental Methods}

\section{Raman Spectroscopy}

Raman spectroscopy was performed on a TSI ProRaman-L, equipped with a $785 \mathrm{~nm}$ laser, fiber optic cable, thermoelectrically-cooled CCD detector $\left(-50^{\circ} \mathrm{C}\right)$, and 0.5 NA objective, which focuses the laser to a single, circular spot $(d=100 \mu \mathrm{m})$. Power output is adjustable across the range of $\sim 5 \mathrm{~mW}-400 \mathrm{~mW}$. The overall dimensions are 9.5” x 7.25" x 5.25" ( $L \times W \times H)$. The entire setup, less the computer interface, is displayed in Figure 4.

For experiments involving only Raman/SERS - without coupling to MS - plasmonic papers were cut with a $3 \mathrm{~mm}$ hole punch (Hobby Lobby, Normal, IL). Punched-out samples were loaded with $1 \mu \mathrm{L}$ of analyte, placed on a gold or aluminum-covered glass slide (to eliminate background), pinned down with a 3D-printed sample holder (Figure 4, inlaid), and then analyzed 
on the sample stage. Using a USB power meter (THORlabs, New Jersey, USA), laser power output was set to either 10 or $15 \mathrm{~mW}$. A syringe pump was attached to a translational knob of the stage, programmed to an inject/withdraw cycle, and left running during Raman acquisitions. This caused the sample to move relative to the laser, such that each spectrum was collected along a $1.5 \mathrm{~mm}$ path instead of from a single spot.

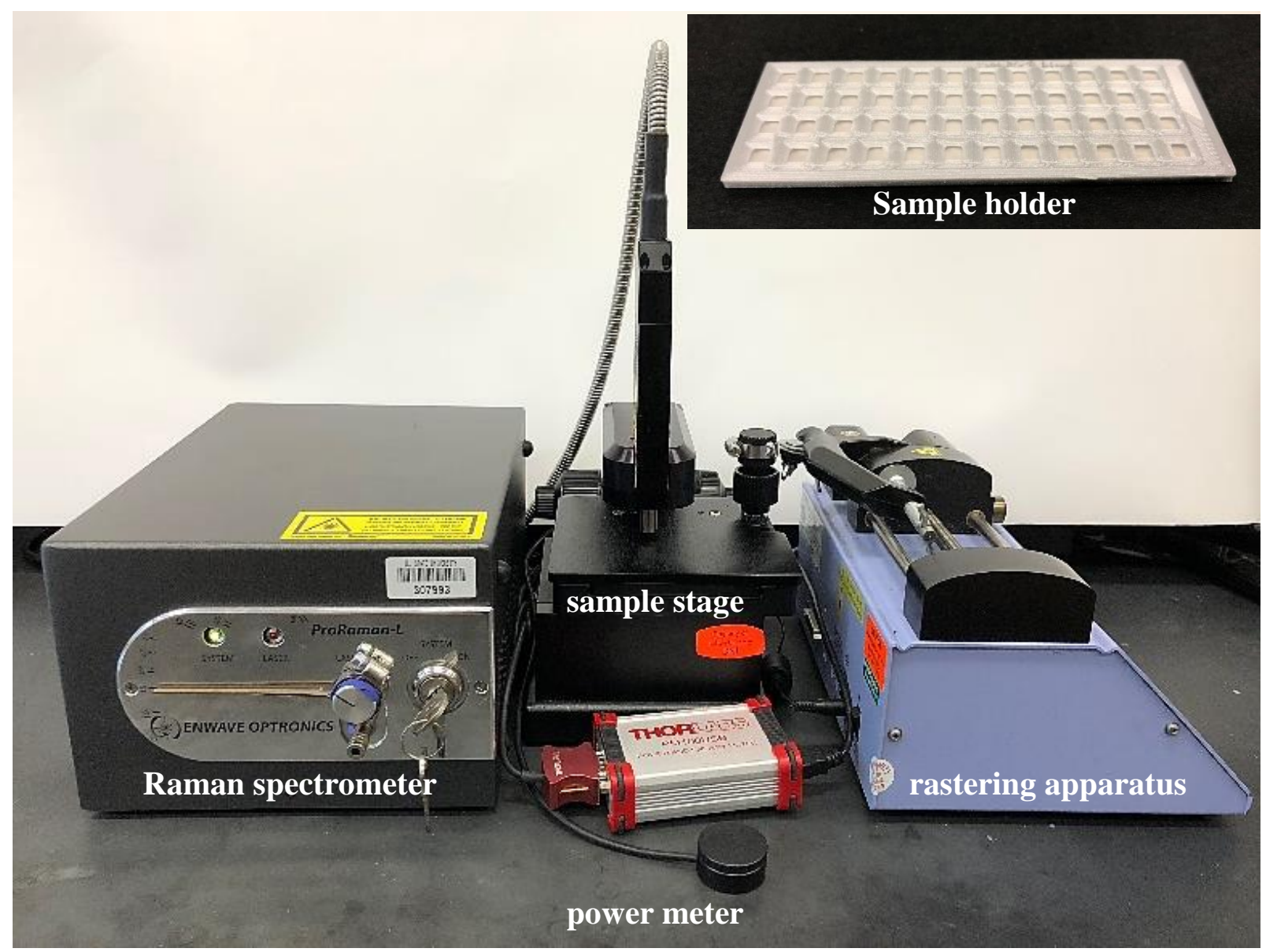

Figure 4. TSI ProRaman-L with attachments. Shown are the power meter, sample stage, and custom rastering apparatus, as well as 3D-printed sample holder (inlaid). The sample holder snaps onto a covered glass slide - each square hole accommodates a $3 \mathrm{~mm}$-punched paper, pinning it down at the edges to ensure it remains at the Raman objective's focal distance. 
When plotted, all Raman spectra were truncated to include only the fingerprint region $\left(400-1800 \mathrm{~cm}^{-1}\right)$. Additionally, in many cases spectra were normalized or offset for the sake of comparison. The former was accomplished by scaling the dominant peak in the fingerprint region of each spectrum to a value of one.

\section{Paper Spray Ionization Mass Spectrometry}

Mass spectrometry was performed on a portable FLIR Systems AI-MS 1.2 (Figure 5), equipped with a cylindrical ion trap (CIT) mass analyzer and tandem MS capabilities. The instrument operates with unit resolution across the mass range $0-4000 \mathrm{~m} / \mathrm{z}$. The overall dimensions are 24" x 20" x 15 " $(L \times W \times H)$, with a weight of 98 lbs.

Plasmonic papers were loosely pinned down atop a grid of isosceles triangles $(b \times h=8$ $\mathrm{mm} \times 9 \mathrm{~mm}$ ). A scalpel was then used to trace along the grid. The resulting triangular papers were separated and placed on glass slides. One $\mu \mathrm{L}$ of drug analyte was pipetted onto individual papers, which were then dried in room air. Samples were then placed in the PSI source.

In the source, papers were held with a piece of copper, which maintained a $+4 \mathrm{kV}$ potential relative to the inlet capillary. Precluding the formation of an electrospray, $5 \mu \mathrm{L}$ of spray solvent was pipetted onto the paper. This solvent consisted of one of two solutions: $\mathrm{MeOH}$ and $\mathrm{H}_{2} \mathrm{O}$ (50:50) with $0.1 \%$ formic acid (v/v) - or acetonitrile and water $(9: 1)$ with $0.1 \%$ formic acid (v/v). Once a stable spray was established, an MS base scan was collected, followed by tandem MS scans as needed. 


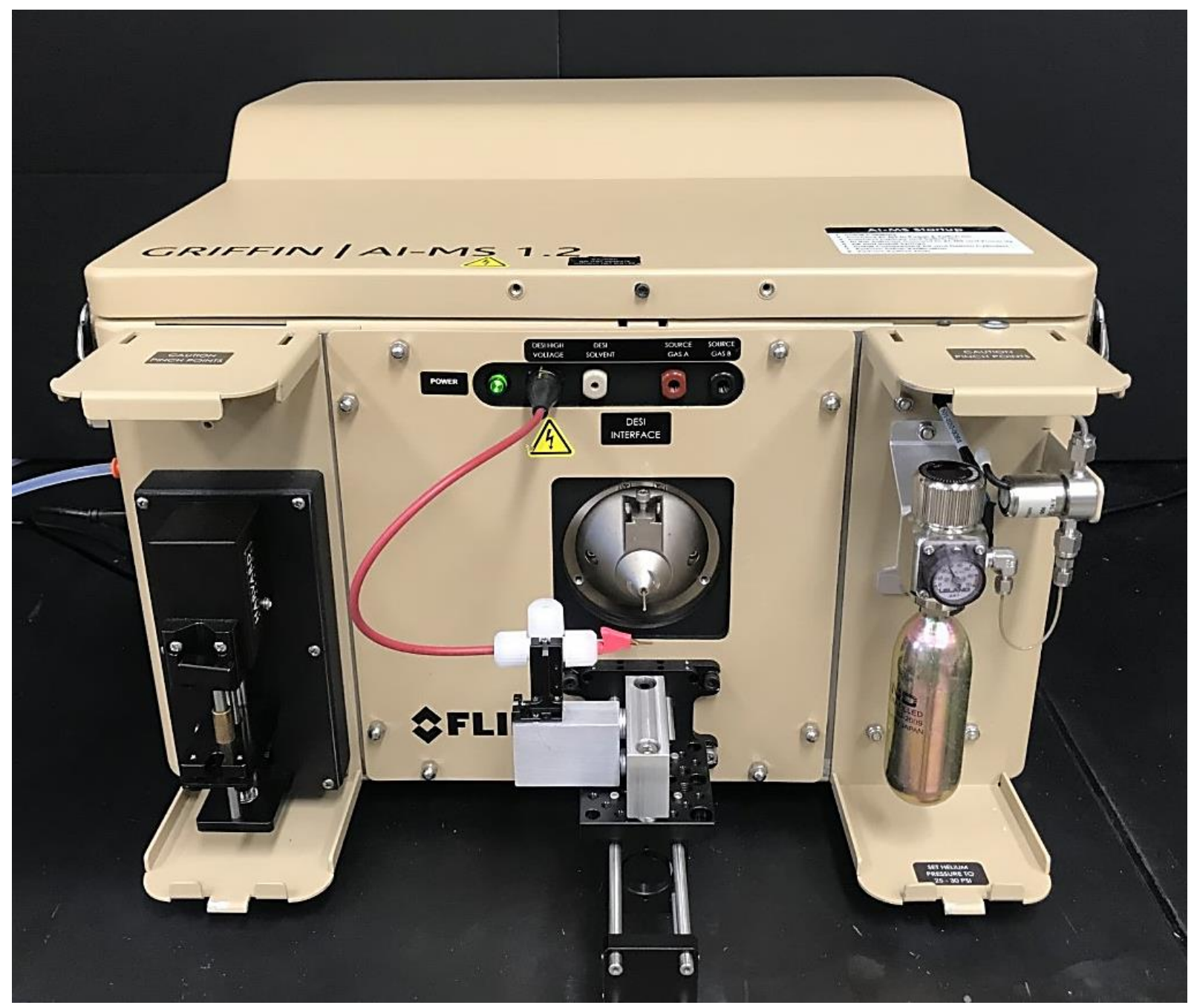

Figure 5. FLIR Systems Griffin AI-MS 1.2, with attached Thorlabs optomechanical cage system. This cage system has been used to accommodate several ionization sources, ${ }^{37}$ and served as a scaffold for integrating the Raman spectrometer and a PSI source in the present work. 


\section{Integrated SERS-PSI-MS}

Method development and staging. Samples were prepared as individual triangles loaded with $1 \mu \mathrm{L}$ samples of one of the five representative drug compounds. Raman spectra were collected before, during, and after the paper spray process to probe any effects the instruments might have on one other. The outcomes of these studies lent themselves to design considerations for the SERS-PSI-MS stage.

The custom-built stage (Figure 6) was constructed using 3D-printed devices (Ultimaker $2+$ ) and optomechanical assemblies (THORLabs, New Jersey, USA) - the latter of which allowed for fine ( $\mu \mathrm{m}$-scale) adjustments in the vertical and translational axes. Each paper sample was placed in a 3D-printed, polypropylene strip with an embedded ribbon of copper foil. This strip was inserted into a channel within the stage, where it could be moved forward or backward relative to the MS.
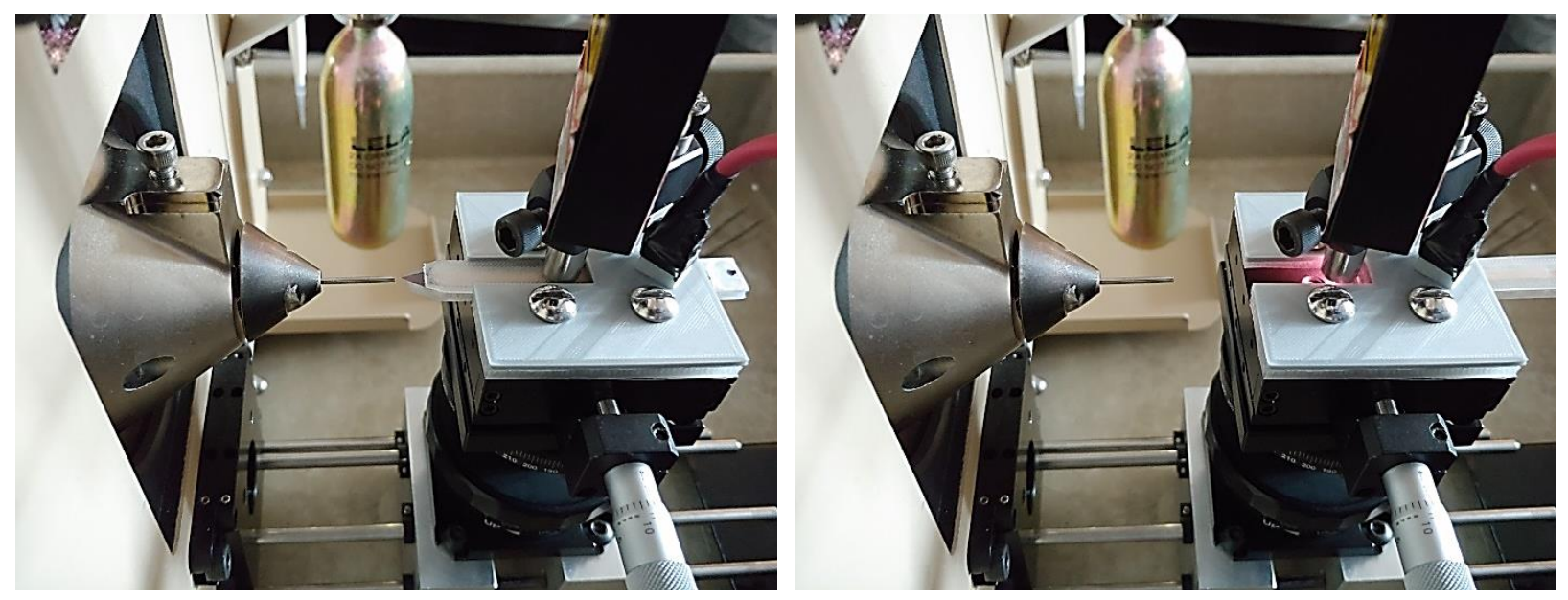

Figure 6. Precision sample stage, supplemented with 3D-printed devices, for integrated SERS-

PSI-MS analysis. At left, the sample strip is slid forward (relative to the MS inlet), initiating electrical contact and - upon addition of solvent - starting the paper spray process. At right, the sample strip is slid backward for collection of Raman spectra. 
Standard operating procedure. For operation, triangularly-cut plasmonic papers - onto which analyte had been deposited and dried - were placed within the polypropylene strips. Strips were then inserted into the channel of the stage, where they could be slid forward or backward. In the forward position, electrical contact was formed for PSI-MS. Five $\mu \mathrm{L}$ of $\mathrm{MeOH}$ : water (50:50) with $0.1 \%$ formic acid was then pipetted onto the paper, initiating paper spray. Minor positional adjustments were made as needed to stabilize the spray and maximize signal. In the backward position, the lights were dimmed for collection of Raman spectra. The initial protocol was to conduct Raman (nondestructive method) prior to MS (destructive method). However, it was found that the quality of Raman spectra greatly improved after PSI-MS - perhaps due to concentration of analyte at the paper tip. The final standard operating procedure reflected this, such that Raman acquisitions always followed PSI-MS.

\section{SERS Identification of Drugs}

Five controlled substances (Figure 7) were chosen to represent common and emerging drug classes (e.g., new psychoactive substances, traditional drugs of abuse, synthetic opioids, abused pharmaceuticals, and synthetic cannabinoids). Sections of plasmonic paper were cut out with a $3 \mathrm{~mm}$ hole punch (Hobby Lobby, Normal, IL). In order to probe the potential of SERS for detecting these substances, $1 \mu \mathrm{g}$ of each was deposited (as $1 \mu \mathrm{L}$ of $1 \mathrm{mg} / \mathrm{mL}$ standard) on three respective paper punches. Deposition - rather than soaking, which is also common in SERS literature - was chosen to mirror the potential end-use of plasmonic paper as swabs. One sample of each drug was also prepared on a hole punched from an unmodified Whatman filter paper. All of these were dried in air for a minimum of $5 \mathrm{~min}$, then loaded into the sample holder. At this point, Raman spectra were collected while adjusting parameters of laser power, acquisition time, and extent of averaging. 
For the optimized collection of Raman spectra, power output was set to $10 \mathrm{~mW}$. Spectra were collected from three areas on each paper punch, while using the previously described rastering device. Each spectrum was recorded as the average of three $10 \mathrm{~s}$ acquisitions.

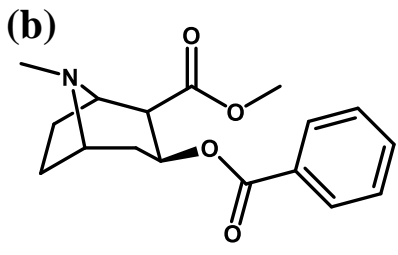

(d)

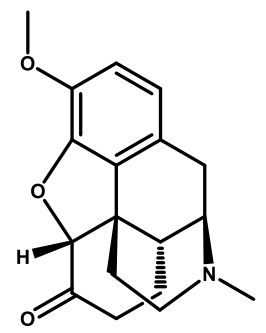

(a)<smiles>COc1cc(CCN)c(OC)cc1Br</smiles>

(c)

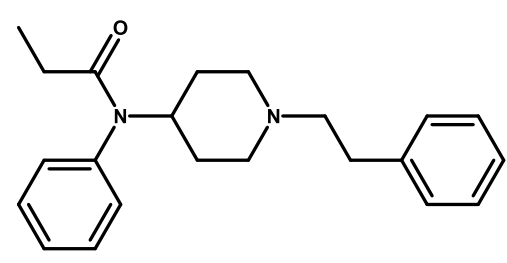

(e)

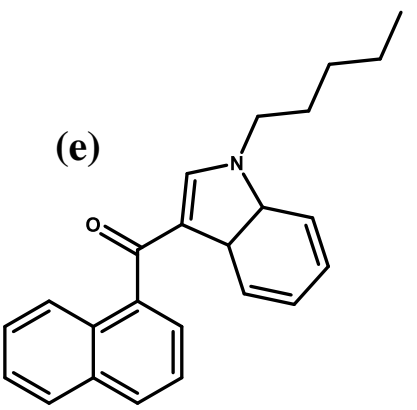

Figure 7. Representative drug compounds used in this study. 2C-B (a), cocaine (b), fentanyl

(c), hydrocodone (d), and JWH-018 (e), chosen to represent several classes of controlled substances: phenethylamines, traditional drugs of abuse, synthetic opioids, abused pharmaceuticals, and synthetic cannabinoids, respectively.

\section{SERS Limits of Detection}

Calibration curves were generated for SERS response of each of the five drugs on plasmonic paper. Five serial dilutions, each with a 1:4 dilution factor, were prepared from the 1 $\mathrm{mg} / \mathrm{mL}$ stock solutions. Spectra were taken after the addition of $1 \mu \mathrm{L}$ methanol, then after the sequential additions of $1 \mu \mathrm{L}$ from each dilution, beginning with the lowest concentration. Thus, the amount of deposited drug progressed from $\sim 1 \mathrm{ng}$ to $\sim 1.3 \mu \mathrm{g}$. The experiment used three separately prepared plasmonic papers - fifteen paper punches were taken from each paper (three for each drug). Each SERS spectrum was collected as the average of three $10 \mathrm{~s}$ integrations, with 
the laser output set to $10 \mathrm{~mW}$. Three spectra were collected per paper punch, with the laser rastering during acquisitions. To construct the plots, the intensity of a prominent, analyte-specific peak was plotted as a function of the total amount of deposited drug.

\section{Density Functional Theory: Calculated Spectra}

Averaged spectra of $333 \mathrm{ng}$ of deposited drug, besides their role in LOD calculations, were also considered separately for comparison to simulated spectra. These were calculated using density functional theory (DFT). All calculations were performed in Gaussian 2009, using the B3LYP functional and with the basis set $6-311++\mathrm{G}(\mathrm{d}, \mathrm{p})$. Calculated frequencies were scaled by a factor of $0.9679,{ }^{90}$ then offset from the SERS spectra for comparison.

\section{Discrimination among Isomers Using SERS}

Two sets of isomers were selected, which had been demonstrated to be indistinguishable by MS and tandem MS: three isomers of the synthetic cannabinoid JWH-018, as well as two tryptamine derivatives - psilocin and bufotenine (from psychedelic mushrooms and the genus species toad, respectively). Chemical structures of these compounds are shown in Figure 8.

Spectra were collected as in the previous experiment, from three regions of each paper punch, after the addition of $1 \mu \mathrm{g}$ drug. Each isomer was represented on nine total punches (taken from across three individually prepared plasmonic papers). DFT spectra were calculated for each drug compound, using the same parameters as previously noted. 
(a)

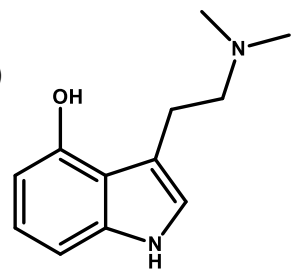

(b)

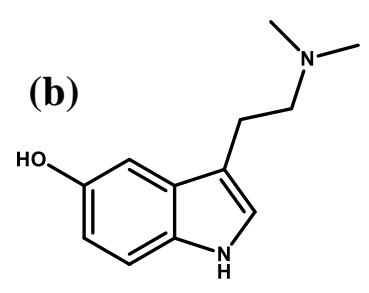

(c)

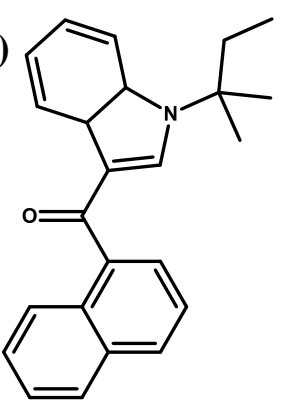

(d)

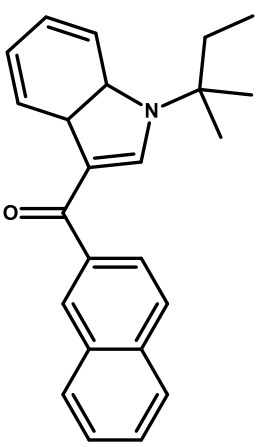

(e)

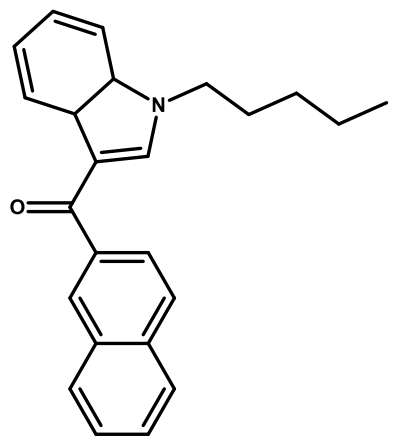

Figure 8. Structural and positional isomers selected to assess SERS discriminating power. The two sets utilized were the tryptamine derivatives psilocin (a) and bufotenine (b), as well as compounds related to JWH-018: N-1,1-dimethylpropyl (c), 2'-naphthyl (d), and 2'-naphthylN-1,1-dimethylpropyl (e) isomers.

\section{SERS-PSI-MS Error-rate Study}

\section{Method Validation}

The previously outlined procedure for collecting SERS after PSI-MS on the custom stage was evaluated in a series of experiments, which included a mock error-rate trial $(N=50)$. SERS spectra in this trial were collected as the average of six $15 \mathrm{~s}$ acquisitions, with the laser output at $10 \mathrm{~mW}$. Samples were randomly prepared, as negative or with one of the five representative drugs. The amount of applied drug was $50 \mathrm{ng}$ in the case of $2 \mathrm{C}-\mathrm{B}$, cocaine, fentanyl, and hydrocodone - and $200 \mathrm{ng}$ in the case of JWH-018, which had a higher detection limit for both techniques. 


\section{Error-rate Study}

The discriminatory capabilities of the combined SERS-PSI-MS platform were tested in a larger error rate study $(N=500)$. All operating parameters were the same as previously noted, except that the laser output was increased to $15 \mathrm{~mW}$. All samples were randomly prepared in like fashion to the mock trial, with 80 samples of each analyte and 100 negatives. Plasmonic papers were freshly prepared every two days, and samples were prepared and analyzed in 25 sets of 20 samples. A blank was loaded before each sample and analyzed by both methods. Throughout the study, documentation was made for the dates on which plasmonic papers had been prepared, loaded with analytes, and analyzed.

All spectra were visually interpreted by experienced users. When SERS and PSI-MS results did not agree (i.e., an ambiguous result), a second and third test were performed. If either of these retests brought forth further ambiguity, the method was considered to have erred. Variability in SERS performance (intra- and inter-day) was calculated as the percent relative standard deviation (\%RSD) of a selected peak-to-peak ratio. These ratios served as crude approximations of the ability of the plasmonic papers to produce a consistent spectral fingerprint. 


\section{CHAPTER III: RESULTS \& DISCUSSION \\ SERS Detection of Drugs}

\section{Optimizing SERS Sensing on Plasmonic Papers}

The first objective within this work was to optimize the collection of SERS spectra for controlled substances deposited on plasmonic papers. This involved varying the laser power, acquisition time, and extent of sample averaging. Plasmonic papers were used as received from a collaborating laboratory.

Laser power output within the range of $10-15 \mathrm{~mW}$ (for a spot size of $\sim .008 \mathrm{~mm}^{2}$ ) seemed to offer the best balance between maximizing intensity while reducing damage to the substrate. The latter issue is seen in changes to the background features when the output was reached 20 $\mathrm{mW}$ (Figure 9). Furthermore, it was consistently found that acquisition times of at least $10 \mathrm{~s}$ were needed to obtain appreciable signals, while acquisitions lasting longer than $30 \mathrm{~s}$ were deleterious in the case of some drug analytes. This is because of fluorescence contributions, which are nearly ubiquitous in Raman spectra. Fluorescence signals are typically removed mathematically; at longer acquisition times, however, fluorescent photons bleach the detector. 


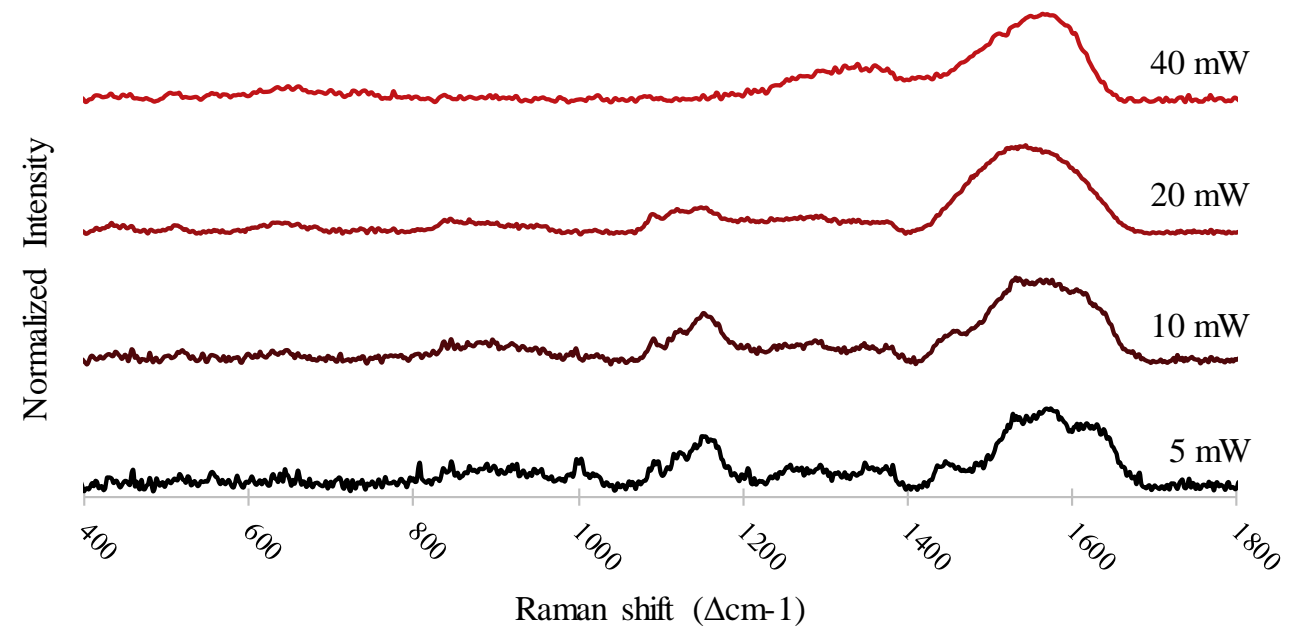

Figure 9. Optimization of power settings for the $785 \mathrm{~nm}$ Raman excitation laser on dry plasmonic paper. The $10 \mathrm{~mW}$ spectrum is similar to the $5 \mathrm{~mW}$ spectrum, but with less noise. At $20 \mathrm{~mW}$ and above, however, the fingerprint begins to change - suggesting undesirable photodegradation.

Averaging spectra has the effect of reducing the noise, scaling it by a factor of $1 / \sqrt{n}$ (where $n=$ number of runs). Efficient noise-suppression can be facilitated by intra-scan averaging, where each recorded spectrum is the average of multiple individual spectra. While extensive averaging is desirable, a decision was made to restrict total collection times to between 30 and 90 $\mathrm{s}$, in order to conform to the rapid analysis desirable in law enforcement settings. Thus, there were typically 3 to 6 acquisitions averaged for each spectrum.

Collection from different spots on the plasmonic paper, even $<1 \mathrm{~mm}$ apart, frequently showed high variability in signal strength. This could presumably be caused in two ways. Firstly, analyte could be unevenly distributed through the paper, as an artifact of pipetting known as the coffee ring effect. ${ }^{91}$ When dissolved analyte is deposited on a surface, it disperses and dries such that the perimeter of the wetted area has higher analyte concentrations than the center. 
The other cause of spot-to-spot variability is the uneven distribution of SERS hotspots - a limitation in the plasmonic papers' preparation. During soaking, the AuNPs adsorb and randomly form aggregates on the cellulose fibers of the paper (Figure 3c). Hot spots result from aggregates that possess the ideal size and geometry (which determine the SPR, as well as the adsorbed species' access to maximum electric field enhancement). However, visual discernment conveys inconsistencies in AuNP loading: many sections on the top-sides of papers are tinted differently, and a view of the bottom-sides reveals that the AuNPs penetrate the paper to different degrees (Figure 10). Since plasmonic papers have been described as three-dimensional enhancing substrates, these inconsistencies could easily sum to the observed signal fluctuations.

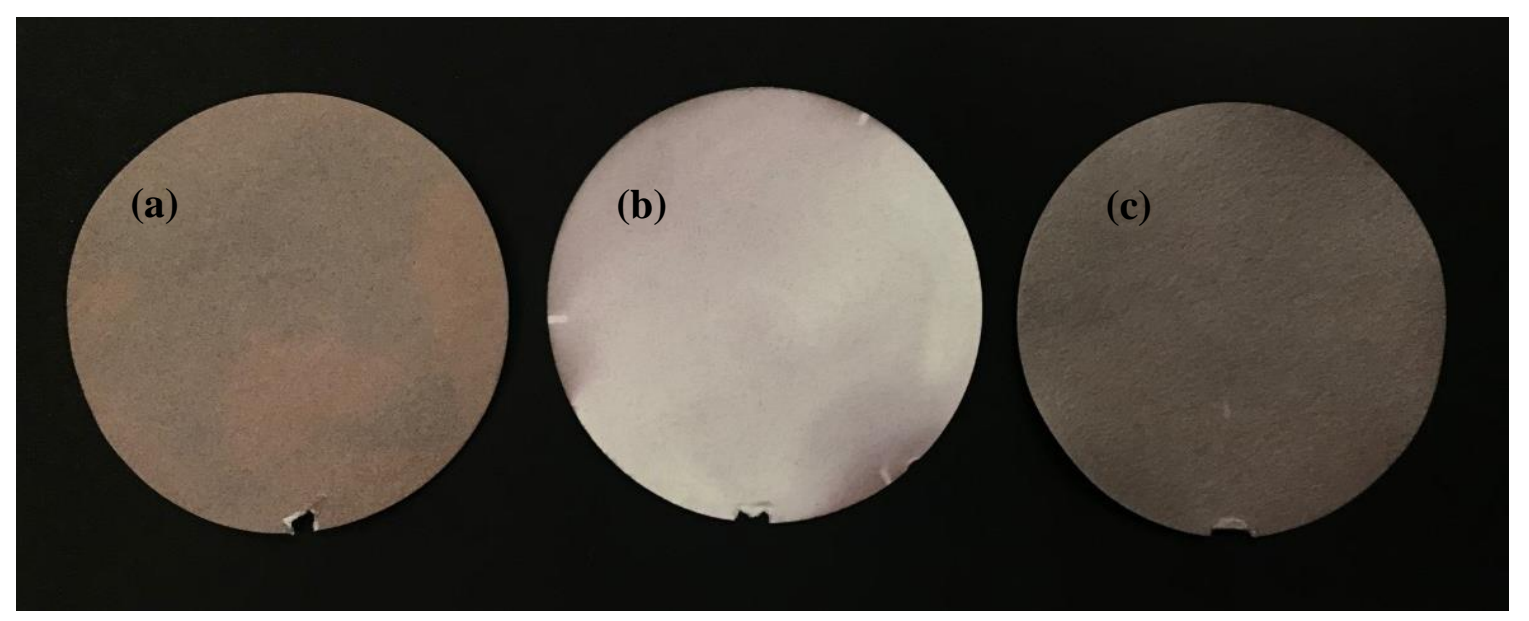

Figure 10. Image capturing the degree of homogeneity in AuNP loading on plasmonic paper, both on the top, sensing side (a), and the bottom side (b). Uniformity can be improved upon with increased experience pipetting colloid over the paper (c), but the aspect of uneven penetration through the paper remains. These differences, along with the coffee ring effect, are believed to be the main source of spot-to-spot variability in SERS signals. 
To minimize the spot-to-spot variations, the volume for additions of sample was set at 1 $\mu \mathrm{L}$ - an amount which would barely saturate a $3 \mathrm{~mm}$-punched paper without welling up or spilling over. Additionally, the previously described rastering apparatus increased the extent of spot-to-spot averaging within each acquisition. As shown in Figure 11, rastering the laser led to increases in signal strength and decreases to the variability among scans - both of which favorably affected the signal-to-noise $(\mathrm{S} / \mathrm{N})$ ratio. These improvements are also promising for the potential to compile a spectral library, in order to eventually identify substances by spectral matching.
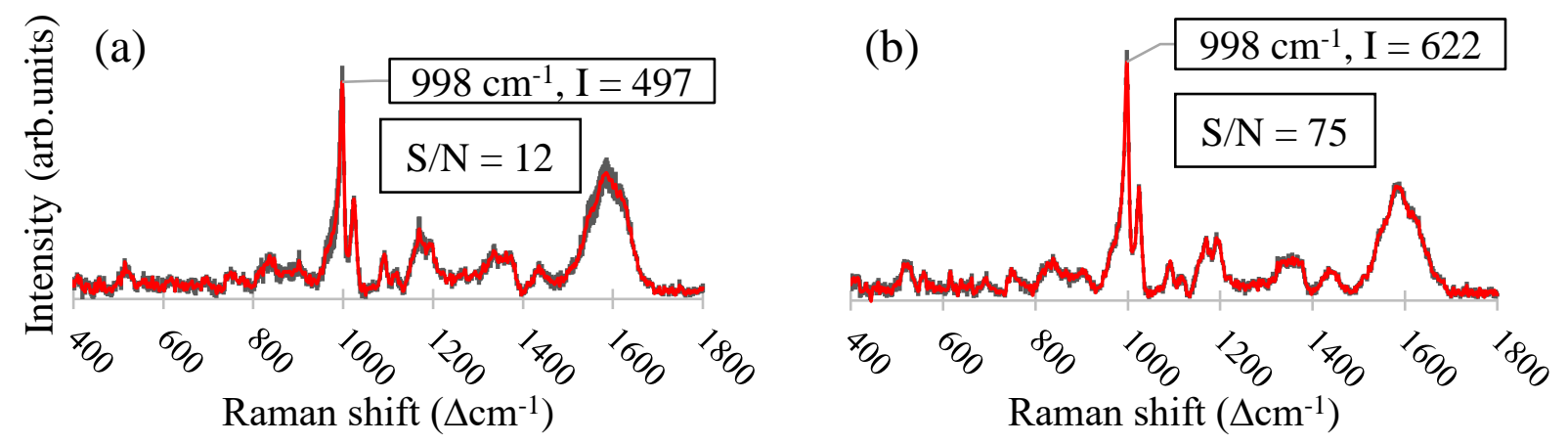

Figure 11. Raman acquisitions collected from a punch of plasmonic paper, loaded with $1 \mu \mathrm{g}$ fentanyl. Spectra were collected from three areas, both with the laser stationary (a) and rastering (b). The $\mathrm{S} / \mathrm{N}$ ratio was calculated by dividing the intensity of the $998 \mathrm{~cm}^{-1}$ peak by the standard deviation of the baseline at its left $\left(940 \mathrm{~cm}^{-1}\right)$. Overall, rastering the laser led to an appreciable increase in the $\mathrm{S} / \mathrm{N}$ ratio.

\section{SERS of Representative Drugs}

Using the optimized collection parameters, Raman spectra were obtained for $1 \mu \mathrm{g}$ of each drug, deposited separately on three punches of plasmonic paper and one punch of unmodified 
filter paper. Spectra were collected in three regions of each punch and averaged together $\left(N_{\text {plasmonic }}=9, N_{\text {unmodified }}=3\right)$. The results are displayed in Figure 12, where each spectrum is normalized and offset to aid in visualization of the different Raman shifts and relative intensities.
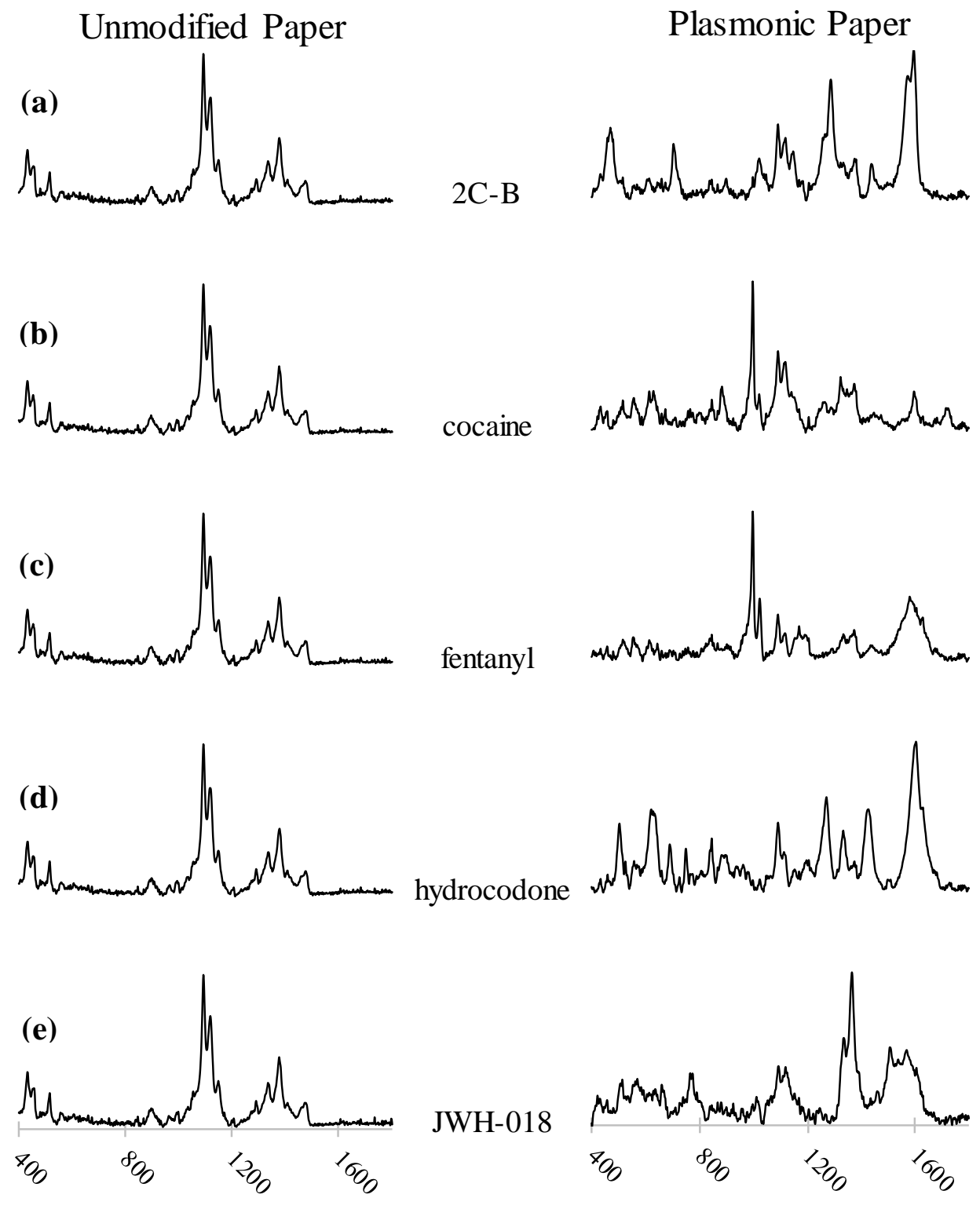

Figure 12. Normalized, offset Raman spectra of $1 \mu \mathrm{g}$ each of 2C-B (a), cocaine (b), fentanyl (c), hydrocodone (d), and JWH-018 (e) on 3 mm-punches of unmodified Whatman filter paper 
(left) and plasmonic paper (right). There are no distinguishing features among the spectra collected from unmodified papers, while plasmonic papers show distinct, analyte-specific Raman shifts.

There are a multitude of peaks exclusive to the respective analytes on plasmonic paper samples, while spectra obtained from analyte applied to unmodified papers are indistinguishable from one another. Each SERS spectrum is distinct, representing a sought-after molecular fingerprint, specific to each drug. In terms of sensitivity, the indication is that $1 \mu \mathrm{g}$ of drug, dispersed through a $3 \mathrm{~mm}$-punched paper, can readily be identified with SERS. If the drug is assumed to be evenly spread within the paper, the amount surveyed by the laser scales to $\sim 1 \mathrm{ng}$ during each acquisition $\left(A_{\text {punch }} / A_{\text {laser spot }} \approx 1000\right)$.

Spot-to-spot and Batch-to-batch Reproducibility

Consistency of performance on the paper SERS substrates was evaluated for each analyte. The results are summarized in Table 3. Figure 13 shows overlaid fentanyl spectra (spotto-spot and batch-to-batch), presented both as absolute and normalized intensities.

\section{Table 3}

Reproducibility of SERS response on plasmonic paper by drug

\begin{tabular}{cccc}
\hline Drug & Peak monitored $\left(\mathbf{c m}^{-\mathbf{1}}\right)$ & Spot-to-spot $(\% \mathbf{R S D})$ & Batch-to-batch (\% RSD) \\
\hline 2C-B & 1292 & $7.6 \%$ & $16.0 \%$ \\
Cocaine & 1000 & $9.5 \%$ & $12.2 \%$ \\
Fentanyl & 1000 & $9.0 \%$ & $16.5 \%$ \\
Hydrocodone & 1272 & $6.0 \%$ & $8.9 \%$ \\
JWH-018 & 1334 & $6.8 \%$ & $7.9 \%$ \\
\hline
\end{tabular}



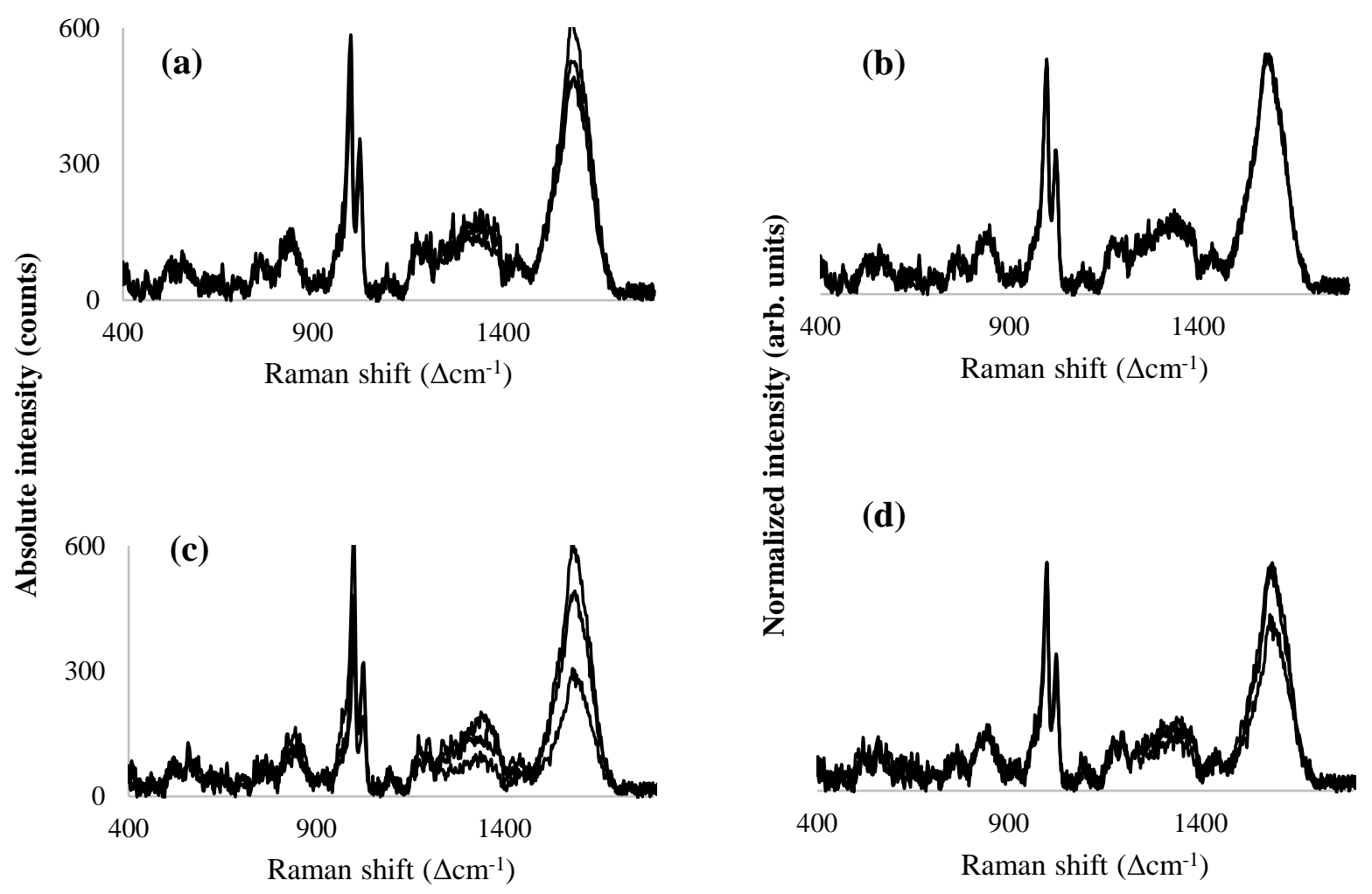

Figure 13. Overlaid SERS spectra for $333 \mathrm{ng}$ of fentanyl, collected from different locations on a single plasmonic substrate (a, b) and three independently prepared substrates (c, d). Spectra at left display the absolute counts for each spectrum, while the same spectra are normalized at right, clearly indicating that - though different spots and substrates yield different degrees of enhancement - the analyte-specific relative intensities are very consistent.

For all analytes, there is an exceptional spot-to-spot reproducibility, with \%RSD $<10 \%$. As previously discussed, the rastering apparatus was used in these acquisitions - this has been shown to bs a key factor for the spot-to-spot performance. The batch-to-batch variations, though higher, are not prohibitive. Perhaps most significantly, however, normalizing spectra makes them nearly superimposable. This makes the consistent performance of a library-matching system 
more likely, and - if an appropriate internal standard is included - does not compromise the potential for quantitative detection.

\section{Limits of Detection}

Sequential additions of drug to punches of freshly prepared plasmonic papers were evaluated by SERS to construct calibration curves. Three papers were utilized, which had all been separately prepared from the same, fresh batch of colloidal AuNPs. Limits of detection (LODs) were calculated by plotting the intensity of a single, prominent analyte-specific peak as a function of the amount of deposited drug analyte - a logarithmic equation was then used to fit each plot. The LODs are presented in Table 4, alongside those from plasmonic PSI-MS analysis. Spectral data and calibration curves are presented in Figures 14 - 18. Since SERS and PSI-MS experiments were conducted using different sizes of paper, LODs are also presented in a form that takes the area of paper into account $\left(\mathrm{ng} / \mathrm{mm}^{2}\right)$. Overall, the sensitivities of both techniques indicate that coupled SERS-PSI-MS - barring unanticipated incompatibilities - would achieve similar sensitivity, at the level of tens of nanograms.

\section{Table 4}

\section{Limits of detection for each of the representative drugs on plasmonic paper}

\begin{tabular}{ccc}
\hline Drug & LOD $($ SERS $)$ & LOD $($ PSI-MS $)$ \\
\hline 2C-B & $3.8 \mathrm{ng}\left(0.53 \mathrm{ng} / \mathrm{mm}^{2}\right)$ & $25 \mathrm{ng}\left(0.69 \mathrm{ng} / \mathrm{mm}^{2}\right)$ \\
Cocaine & $0.6 \mathrm{ng}\left(0.08 \mathrm{ng} / \mathrm{mm}^{2}\right)$ & $13 \mathrm{ng}\left(0.36 \mathrm{ng} / \mathrm{mm}^{2}\right)$ \\
Fentanyl & $1.0 \mathrm{ng}\left(0.14 \mathrm{ng} / \mathrm{mm}^{2}\right)$ & $10 \mathrm{ng}\left(0.28 \mathrm{ng} / \mathrm{mm}^{2}\right)$ \\
Hydrocodone & $3.7 \mathrm{ng}\left(0.53 \mathrm{ng} / \mathrm{mm}^{2}\right)$ & $20 \mathrm{ng}\left(0.56 \mathrm{ng} / \mathrm{mm}^{2}\right)$ \\
JWH-018 & $26 \mathrm{ng}\left(3.7 \mathrm{ng} / \mathrm{mm}^{2}\right)$ & $130 \mathrm{ng}\left(3.6 \mathrm{ng} / \mathrm{mm}^{2}\right)$ \\
\hline
\end{tabular}


It is common in SERS literature to use the intensity of an analyte's most prominent peak (or the area under it) to calculate limits of detection. However, such a method is applied for compounds (e.g. 4-nitrobenzenethiol, a.k.a. NBT) that are known to readily bind plasmonic surfaces (via strong $\mathrm{Au}-\mathrm{S}$ interactions) and elicit intense Raman scattering (from symmetric $\mathrm{NO}_{2}$ stretching, for example). The analytes in this work do not behave in such an ideal manner, and often compete closely with signals that are already present in the spectrum of blank plasmonic paper. These background signals are quite appreciable across the region of $1200-$ $1700 \mathrm{~cm}^{-1}$ - therefore, the most prominent analyte peaks outside of this range were selected for LOD calculations.

Raman is a bulk technique for which signals scale linearly with the number of molecules being analyzed. SERS operates similarly, yet, signals are expected to plateau as analyte achieves monolayer coverage on the plasmonic surfaces. In a full calibration model for SERS, there is expected to be an S-profile, progressing from below the LOD, to the linear range, and finally the point of signal saturation. Notably, it is common in SERS literature to use log or semi-log plots to extend the linear (empirical) range. ${ }^{62,92}$

All of the calibration curves display a saturation profile, wherein signals begin to plateau after an initial sharp increase. The inlaid plots are well fit by logarithmic functions, though 2C-B is an exception. The fit for $2 \mathrm{C}-\mathrm{B}$ seems confounded by the point at $83 \mathrm{ng}$, which has a $\% \mathrm{RSD}$ of $23 \%$ - among the highest. Hydrocodone, which, like 2C-B, seems to display the S-shaped profile, also has a high degree of variability among the nine scans collected. Perhaps these compounds merit further data collection, especially since exclusion of the $83 \mathrm{ng}$ point results in the appearance of good conformity to the other drugs' calibration profiles. For fentanyl, and perhaps cocaine, there is no deviation from the logarithmic best-fit, and the empirical range 
continues to $1.3 \mu \mathrm{g}$. It was not experimentally feasible to extend the data set: the DEA-exempt samples were $1 \mathrm{mg}$ in $1 \mathrm{~mL}$ solvent, and, deposited in $1 \mu \mathrm{L}$ quantities, this would have been very tedious. JWH-018, obtained as $5 \mathrm{mg}$, could easily be examined in larger quantities. At $10.3 \mu \mathrm{g}$ there was still no plateau from the empirical range. 

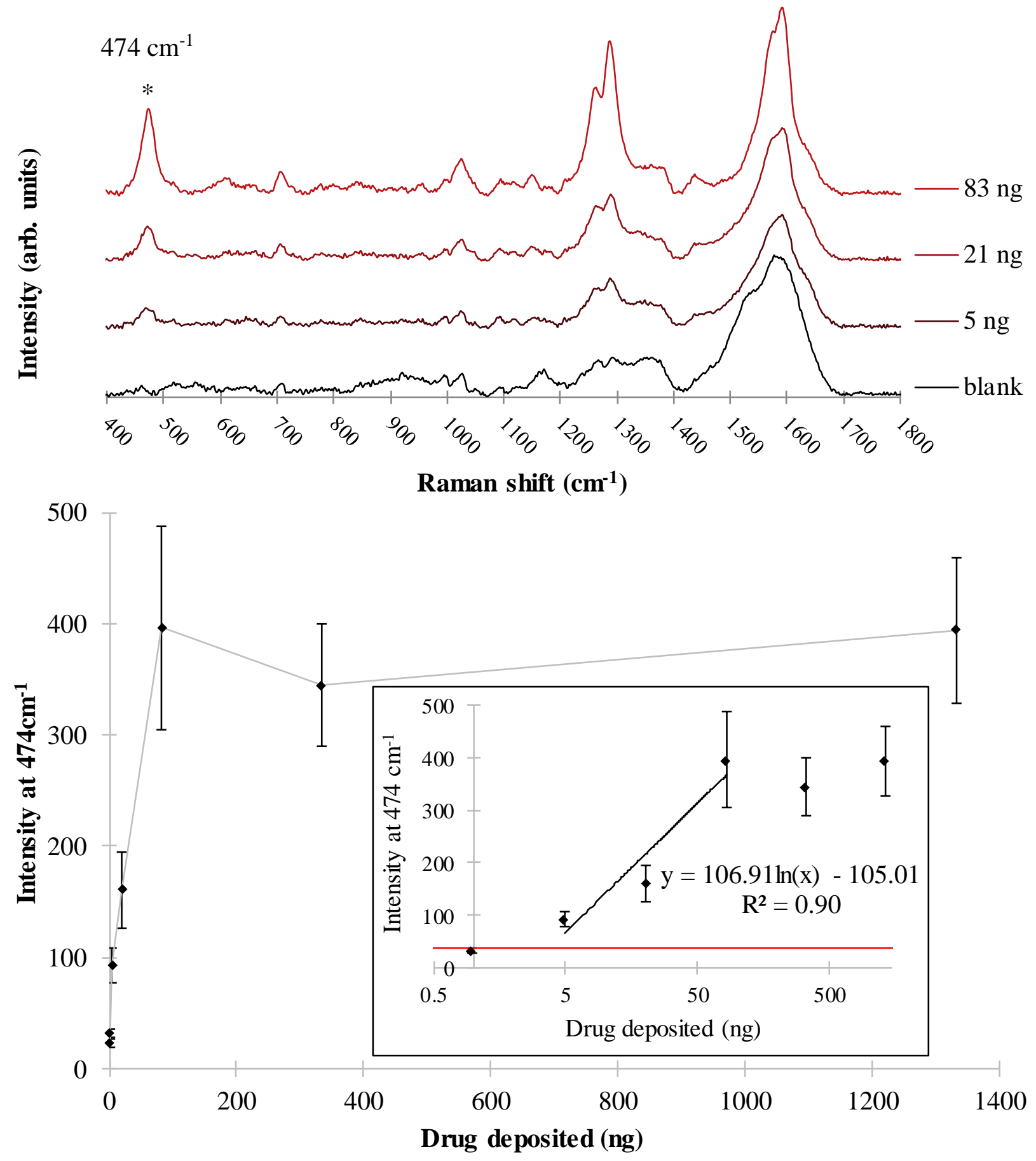

Figure 14. Overlaid SERS spectra and calibration curve for 2C-B. 

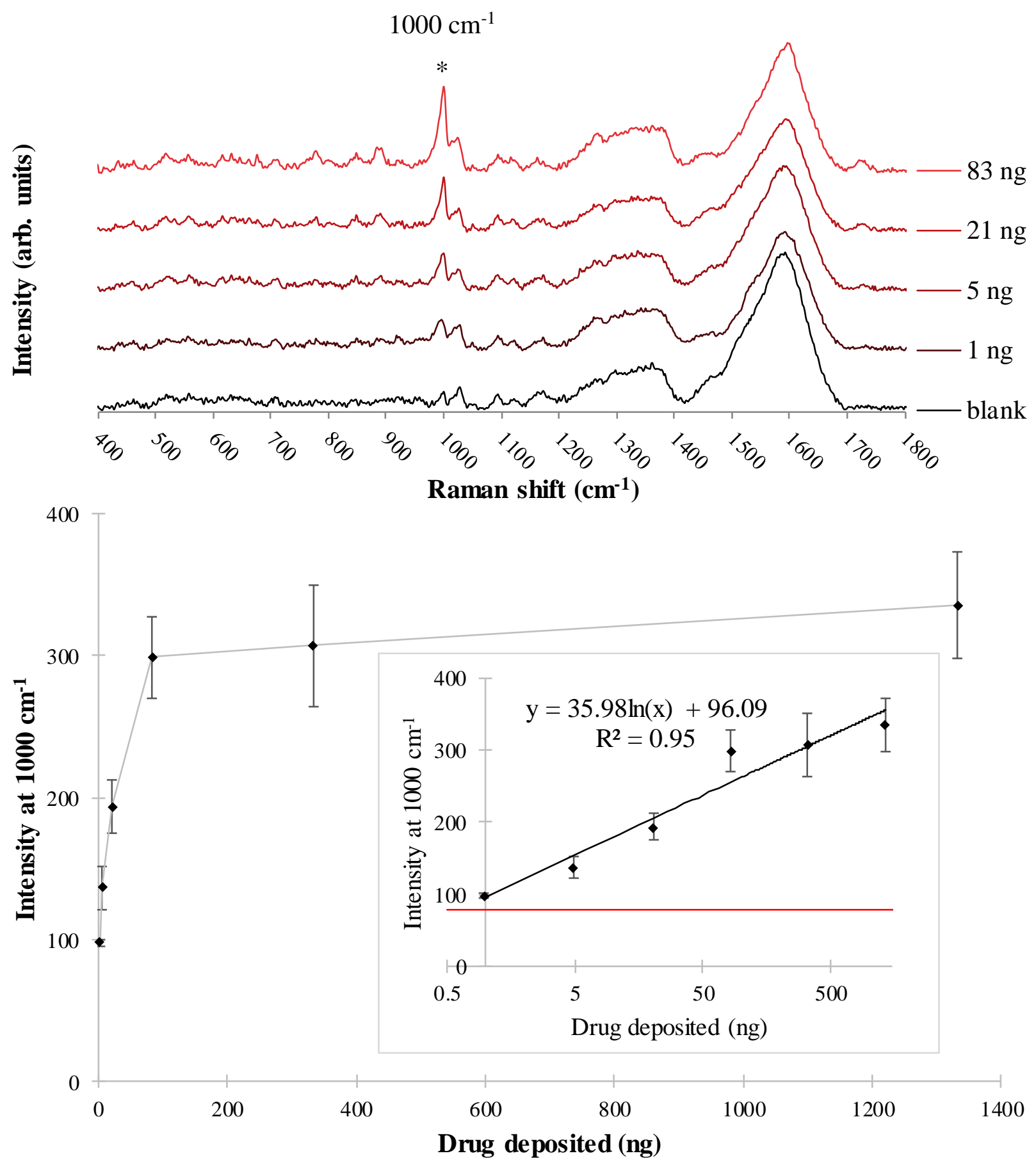

Figure 15. Overlaid SERS spectra and calibration curve for cocaine. 


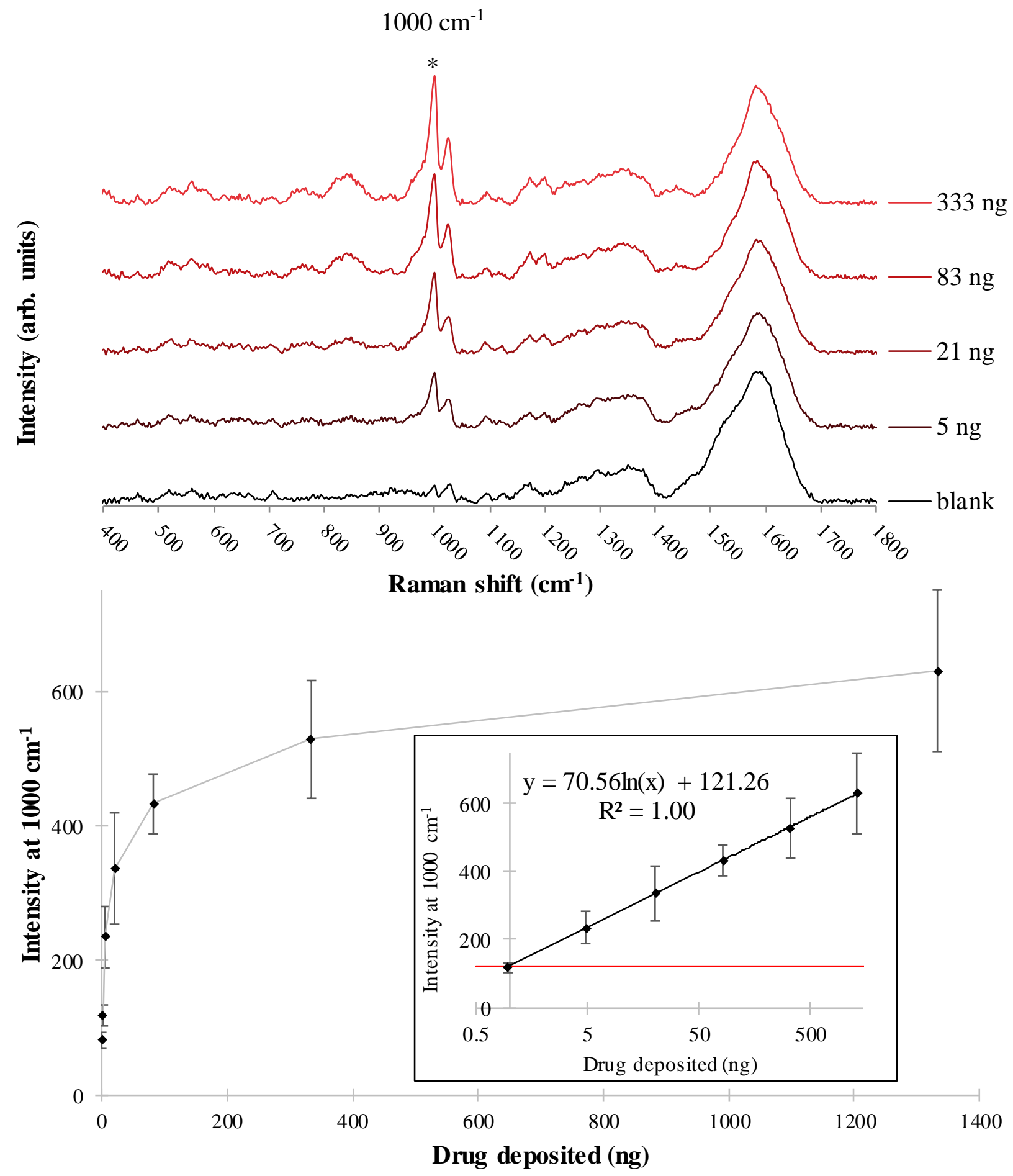

Figure 16. Overlaid SERS spectra and calibration curve for fentanyl. 

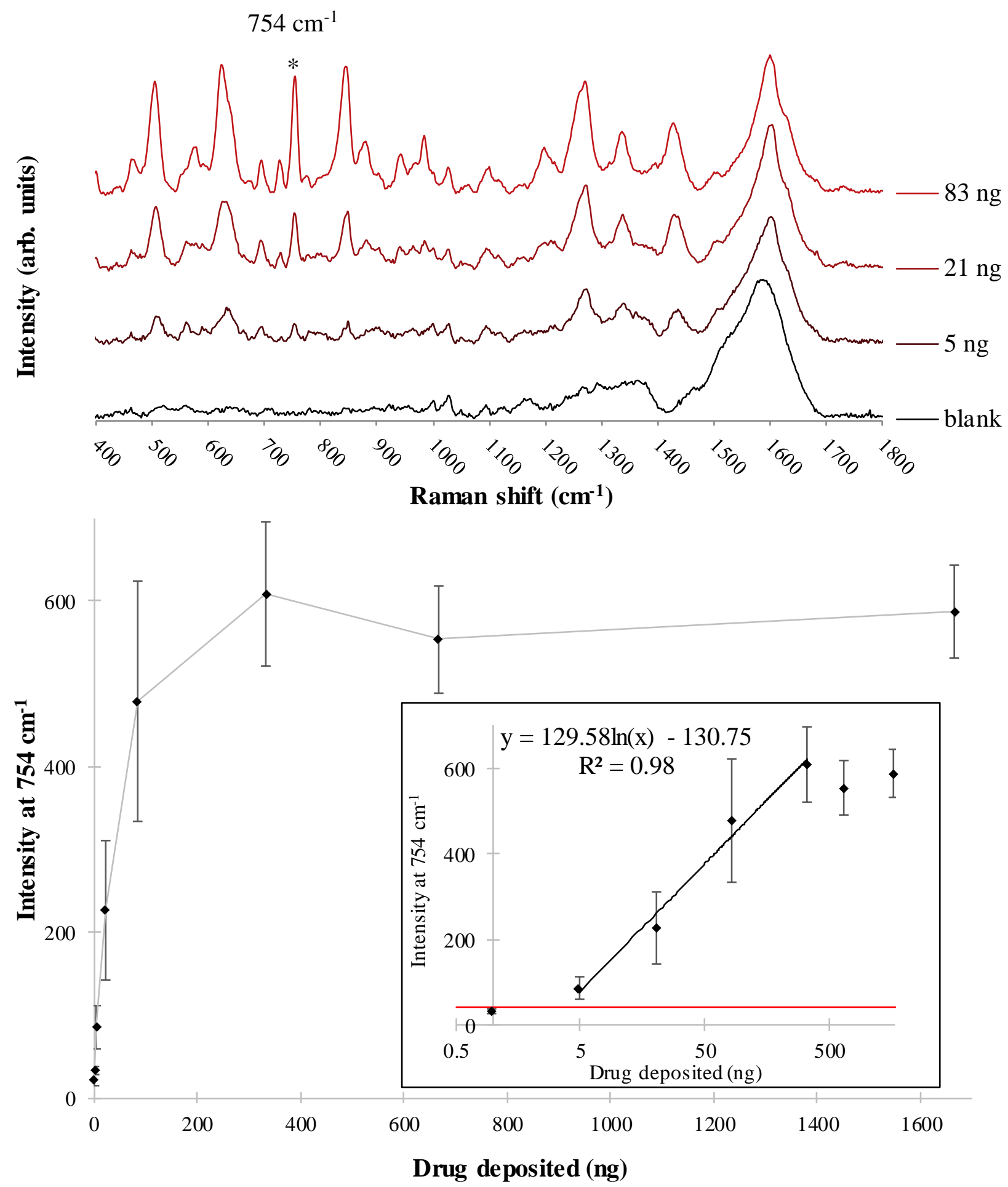

Figure 17. Overlaid SERS spectra and calibration curve for hydrocodone. 

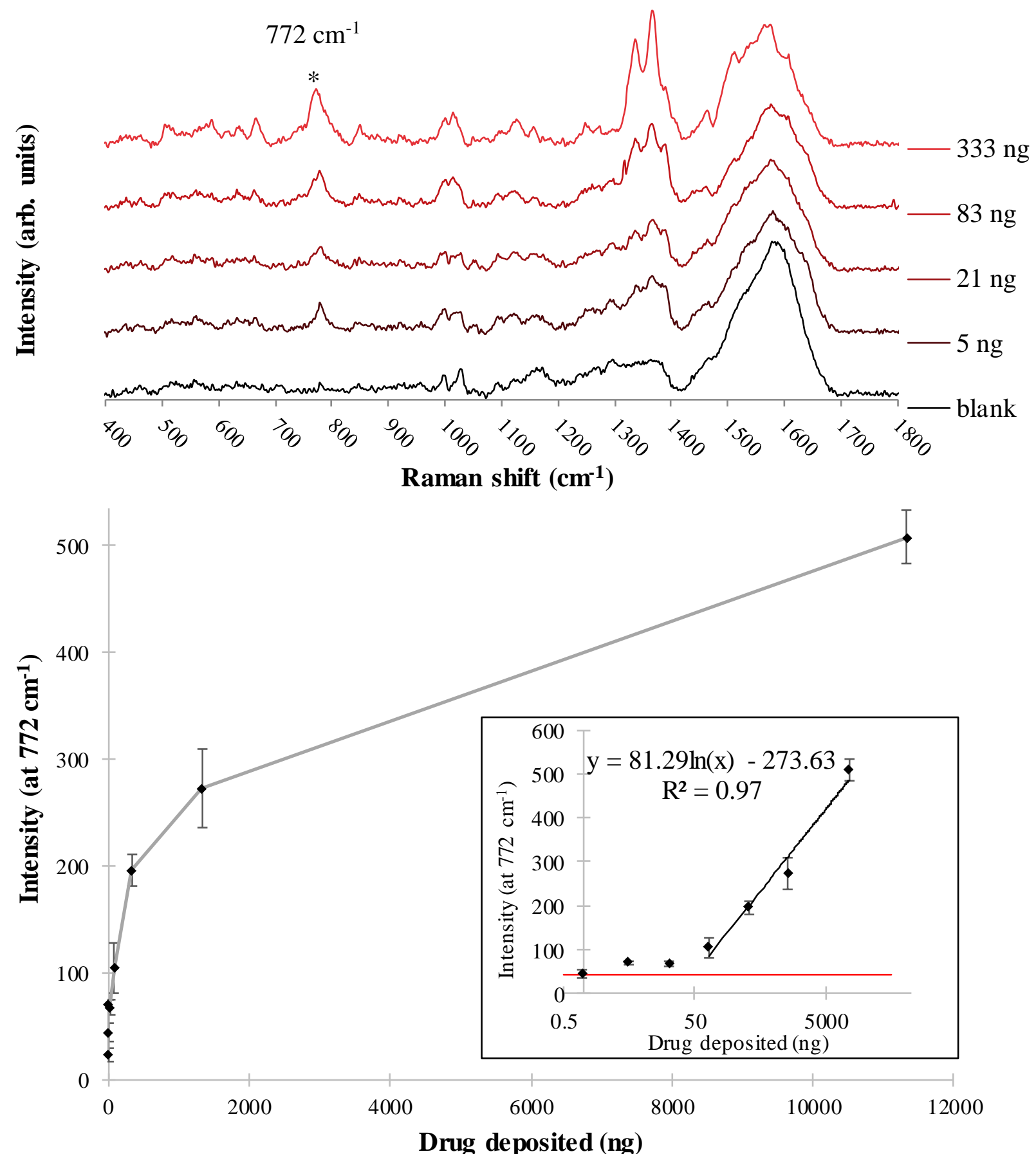

Figure 18. Overlaid SERS spectra and calibration curve for JWH-018.

There are indications for extending the range of these experiments to less than $1 \mathrm{ng}$. Specifically, the LOD for cocaine had to be extrapolated, and none of the compounds failed to 
produce signal at $5 \mathrm{ng}$ (the second-lowest increment examined). Thus, it may be prudent to repeat the experiments, instead initiating depositions of analyte at the level of tens of picograms. However, improving the processing of data by using multivariate statistical tools could prove equally (if not more) useful. ${ }^{62}$ The limitations of a univariate approach to spectral data are delineated below within the context of plasmonic papers' background signals.

There is a complex interplay between background and analyte signals. A model case is shown in Figure 19, using data from 2C-B. At five nanograms, the peak at $1290 \mathrm{~cm}^{-1}$ has grown, while the entire region c.a. 1450 to $1600 \mathrm{~cm}^{-1}$ has been suppressed. Then at $83 \mathrm{ng}$ the $1290 \mathrm{~cm}^{-1}$ peak has continued to rise, and - while the region from 1450 to $1550 \mathrm{~cm}^{-1}$ has continued to decline - analyte-specific peaks from 1550 to $1600 \mathrm{~cm}^{-1}$ have gained prominence. 


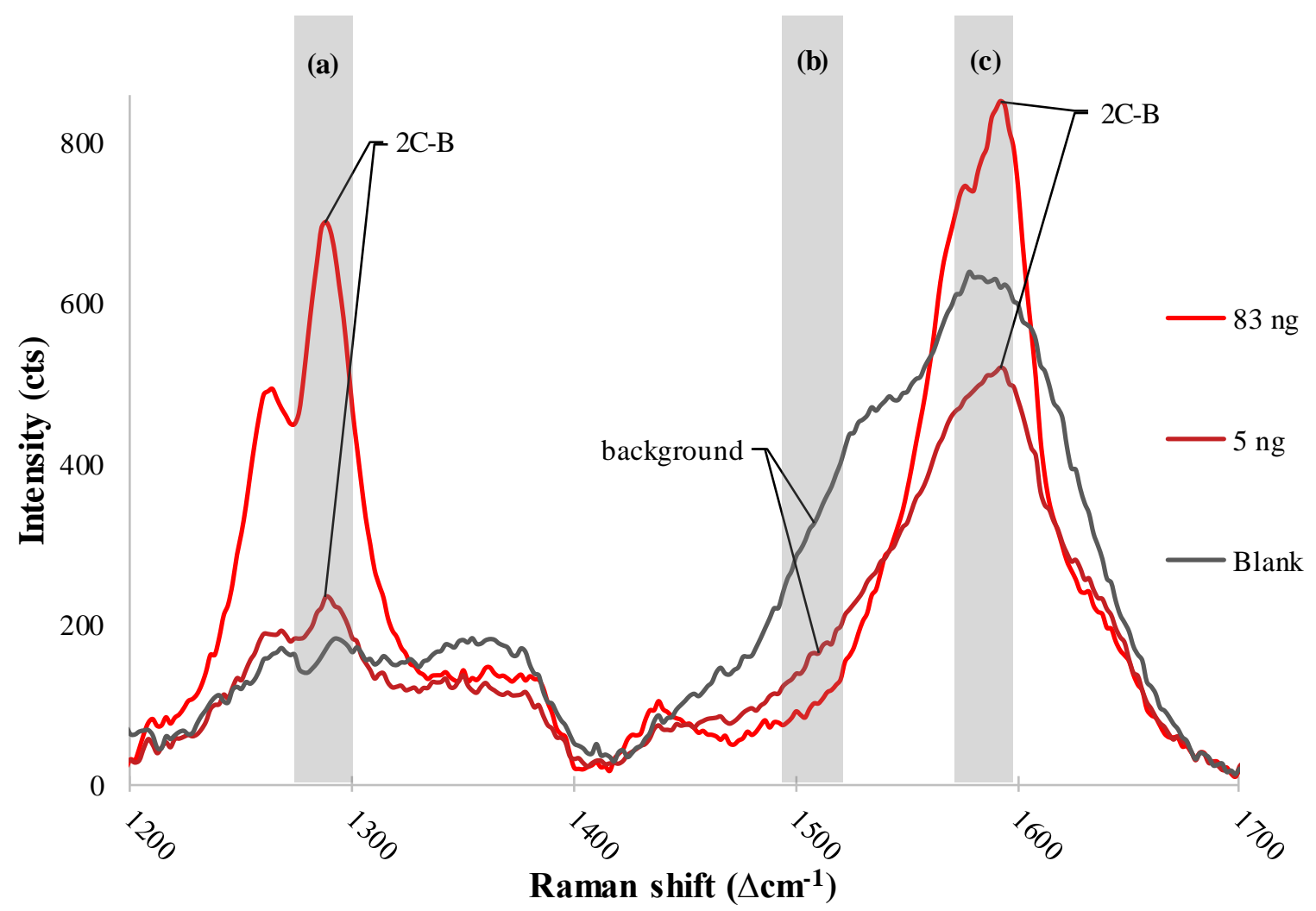

Figure 19. Truncated, overlaid spectra of plasmonic paper with no analyte (grey trace), $5 \mathrm{ng} 2 \mathrm{C}$ B (dark red trace), and $83 \mathrm{ng} 2 \mathrm{C}-\mathrm{B}$ (red trace). show the complicated interplay between background suppression and increasing analyte signal. With additional sample, analyte-specific peaks increase (a), background signals decrease (b), and regions where background and analyte signals overlap decrease, then increase (c). A univariate analysis severely limits the utilization of such trends for quantitative analysis.

There is adequate theoretical basis for these signal fluctuations - non-analyte molecules are present on the surfaces of AuNPs and are gradually displaced by analyte. Citrate is a likely candidate for this initially adsorbed species, since it is used in AuNP synthesis and stabilizes the AuNPs in suspension. Figure 20 shows the outcome of an experiment wherein $1 \mu \mathrm{g}$ of aqueous trisodium citrate was deposited on plasmonic paper. For this amount of analyte, all of the small- 
molecule drug compounds studied showed appreciable spectral fingerprints. However, the relative lack of change in SERS response with addition of citrate supports the notion that it contributes to the Raman fingerprint of the blank (i.e., no analyte) plasmonic paper. Cellulose perhaps also plays a part, since the background shares the profile of some peaks from unmodified paper (e.g. 1290, 1336, and $1376 \mathrm{~cm}^{-1}$ ).

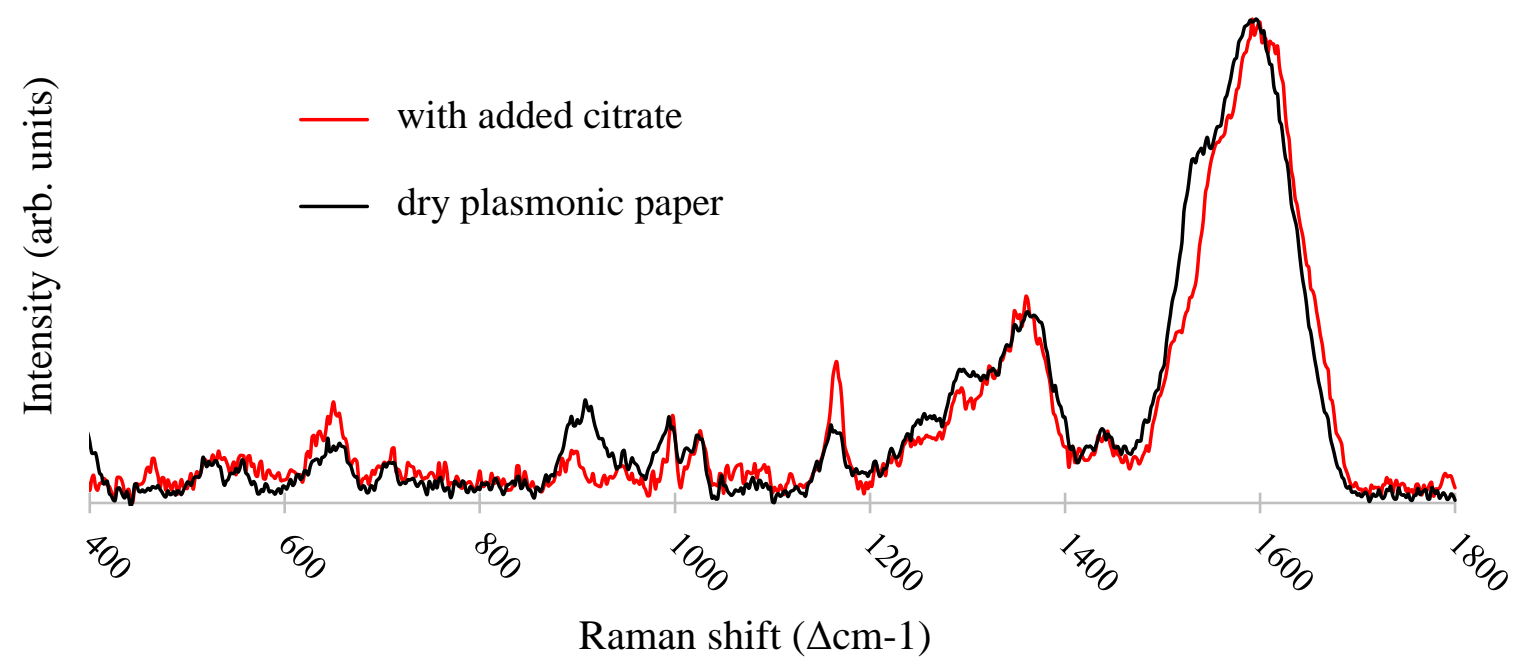

Figure 20. Normalized SERS response of plasmonic paper before and after the deposition of trisodium citrate. The lack of change in the position and relative intensities of peaks suggest that citrate could be responsible for the background signals frequently observed on plasmonic paper.

If background signals were present as sharp, distinct peaks - removed from analyte signals - one of them could be utilized as an internal standard. As seen, this is not the case. This is not a limitation in the data, however, but a limitation in univariate (single-peak) analysis. Using more sophisticated, multivariate statistical tools, several peaks - or the entire spectrum - 
could be utilized. Such tools can deconvolute spectra into the separate contributions of analyte and background species, allowing a comparison between the two for quantitative purposes. Using such means could perhaps correct other disparities in the calibration plots, for example, the onset of signal prior to the calculated LOD for JWH-018.

\section{Conformity to Calculated Spectra}

The accuracy of each drug spectrum was assessed via its conformity to predicted Raman fingerprints. These were calculated using density functional theory (DFT) - a computational tool frequently used in SERS research. DFT describes many types of calculations, all of which arrive at a system's energy quanta as a function of electron density. This is in contrast to the Schrödinger equation $(\hat{\mathrm{H}} \Psi=\mathrm{E} \Psi)$, which arrives at these energy quanta as a particular function (the Hamiltonian operator, $\hat{\mathrm{H}}$ ) of all the positional coordinates for a system's electrons (denoted as $\Psi)$. DFT has the advantage of being more accurate than the simplest approximation of the Schrödinger equation (Hartree-Fock theory). Though corrections to HF greatly improve its accuracy, surpassing that of DFT, these corrections require additional - often prohibitive computational time.

As previously mentioned, DFT calculations were carried out using the functional B3LYP with a $6-311++\mathrm{G}(\mathrm{d}, \mathrm{p})$ basis set, and frequencies were scaled by a factor of 0.9679 - all of these parameters are frequently attested in literature. ${ }^{90,93}$ SERS and DFT spectra for each of the five drugs are normalized and offset in Figure 21. 

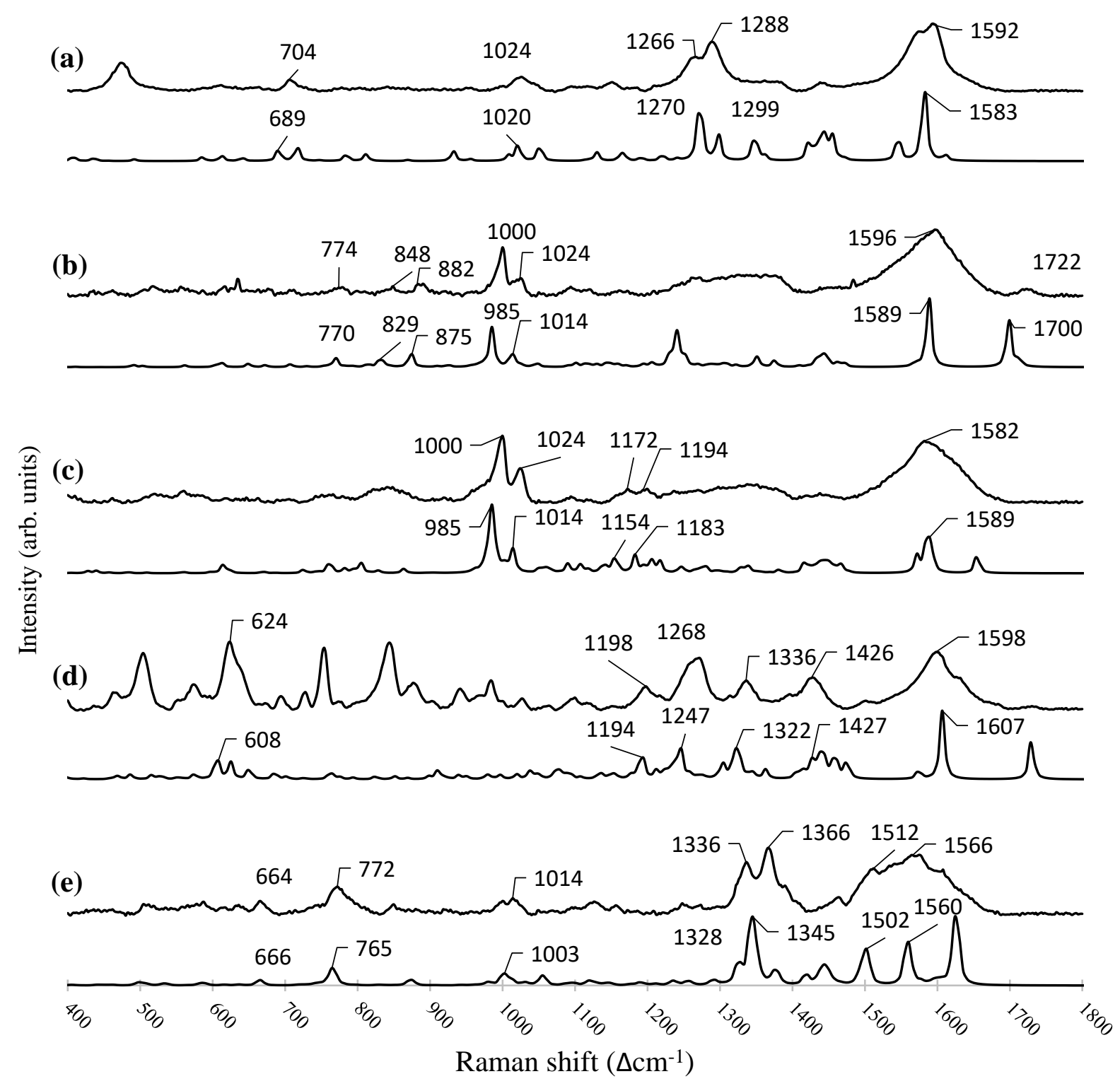

Figure 21. DFT-calculated spectra overlaid with SERS spectra for each drug compound. From top to bottom are 2C-B (a), cocaine (b), fentanyl (c), hydrocodone (d), and JWH-018 (e), with several corresponding peaks labelled for each set. The close agreements further demonstrate the potential for using plasmonic papers to obtain accurate molecular fingerprints for trace levels of analyte. Calculated spectra can also assist in identifying the particular vibrations responsible for each peak in the Raman spectrum. 
The experimental and simulated spectra show very close agreement, with some qualifications. Fluctuations in relative intensities (most pronounced in the case of hydrocodone) are to be expected. This is because the enhancement of a vibration's Raman scattering depends on the proximity and orientation of the pertinent bonds relative to the gold surface. Thus, the way in which molecules are packed on AuNPs causes some vibrational frequencies to be enhanced more than others. Additionally, peaks could be broadened insofar as molecules display a range of orientations on the plasmonic surfaces. Hydrocodone is a rigid, highly conjugated molecule compared with the other analytes it has a lack of freely rotating bonds. This may cause it to adapt a restricted preferred orientation on the AuNP surface, leading to more pronounced alterations in relative intensities.

Another aspect of this divergence pertains to vibrational frequencies. These DFT calculations were performed for single molecules in vacuum. Interactions of the analyte molecules with one another, as well as any tethering of molecules to AuNP surfaces, are unaccounted for. Yet, these interactions can greatly affect the vibrational frequencies; differences between simulated and experimental Raman frequencies are commonly attested. ${ }^{94}$ All this considered, there is excellent agreement between the DFT-calculated and SERS spectra. Nearly all the major peaks predicted in the former are present within $20 \mathrm{~cm}^{-1}$ in the latter.

\section{SERS Discrimination of Isomers}

Accurate identification of isomeric compounds is difficult in MS. Even when equipped with tandem MS capabilities, a mass spectrometer cannot distinguish between species with similar fragmentation patterns. Raman/SERS, on the other hand, derives signal from the unique vibrational energy quanta of molecules. Thus, changes in a functional group's position or 
rearrangement of alkyl chains can be readily identified. The respective MS and Raman/SERS analyses of the isomers examined in this work are shown in Figures 22 and 23.

(a)

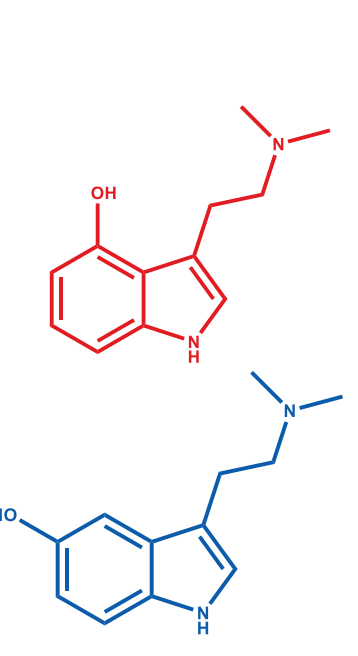

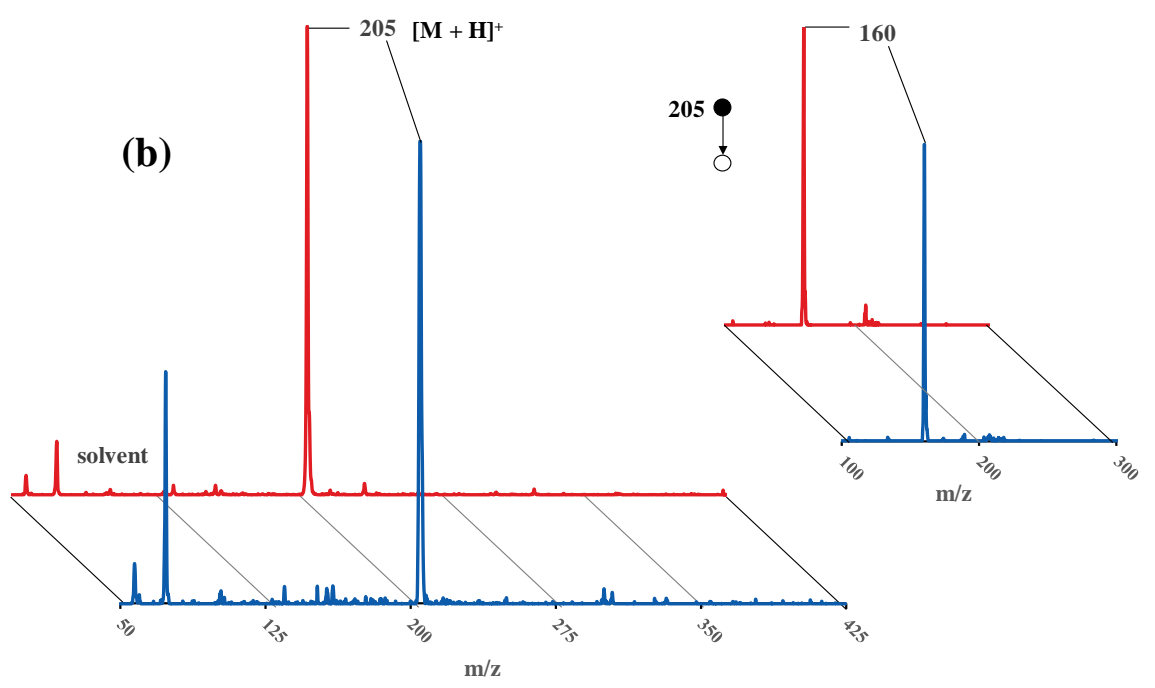

(c)

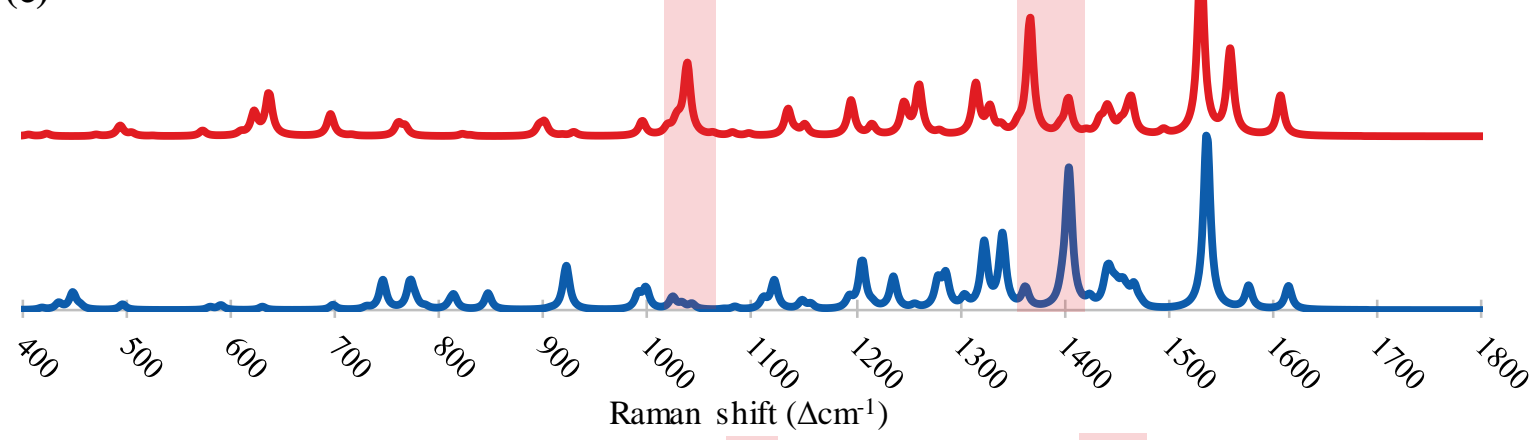

(d)

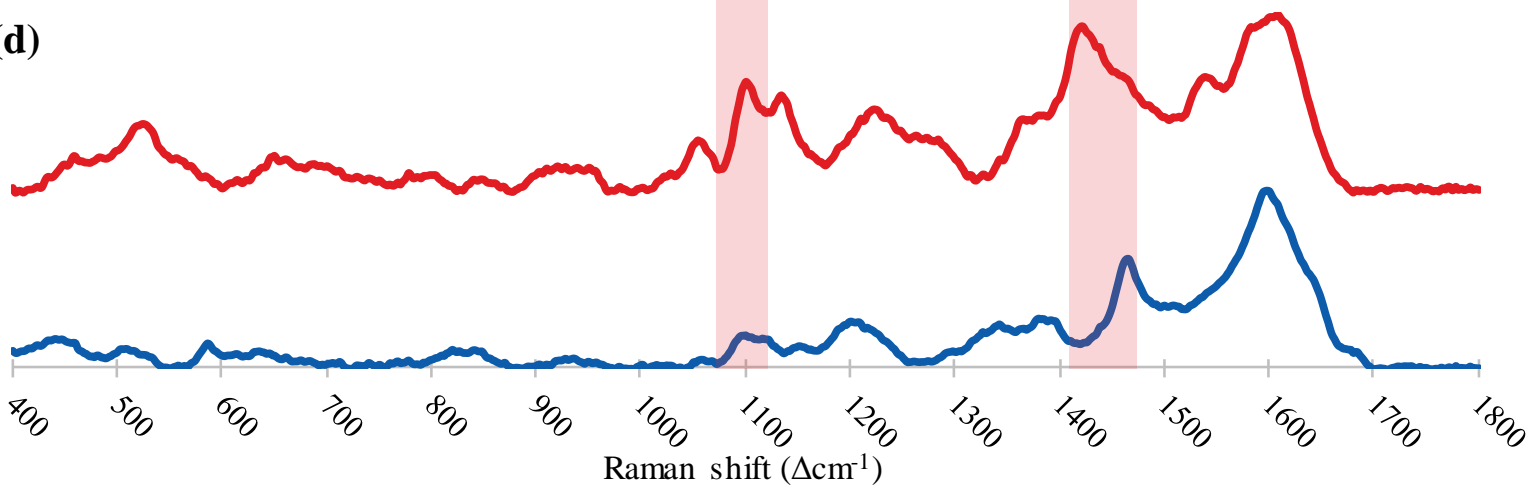

Figure 22. The tryptamine derivatives psilocin (red) and bufotenine (blue) (a). MS and tandem MS analyses (b) provide no distinction between the two. While the overlap between DFT (c) 
and SERS spectra (d) is not very strong, discrimination between the two compounds is facile in either case. The regions highlighted in red reflect some possible similarities between the predicted and experimental fingerprints.

Psilocin and bufotenine are structural isomers where the hydroxyl group occupies different positions on the benzene ring (Figure 22a). The mass spectra of psilocin and bufotenine are superimposable, showing only the protonated molecule at $205 \mathrm{~m} / \mathrm{z}$ (Figure 22b). The same is true for the tandem mass spectra, which show a single peak at $160 \mathrm{~m} / \mathrm{z}$, corresponding to the loss of the dimethylamine fragment (Figure 22b). The DFT spectra predict a facile distinction (Figure 22c), though the accuracy of the simulated spectra is inferior to those of the five compounds previously surveyed (Figure 21). Despite these inaccuracies, however, the experimental SERS spectra (Figure 22d) are repeatably easy to distinguish $(N=9$, across three independent substrates). The differing vibrational frequencies, the ways in which psilocin and bufotenine pack onto the plasmonic surfaces, and perhaps the preferred orientations of each molecule (only psilocin can form internal hydrogen-bonds) give rise to very distinct SERS fingerprints, with some regions - highlighted in red - resembling the calculated fingerprints. The relatively broad SERS peaks could be attributable, as previously mentioned, to a distribution of the molecules' orientations on the gold surfaces.

For JWH-018 and its isomers (Figure 23), MS and tandem MS spectra again show no distinctions (Figure 23b). There are, however, different DFT and SERS spectra for each compound (Figure 23c, d). Some of these differences can be attributed to specific structural elements, as in the case of the 1'-naphthyl (grey, black) versus 2'-naphthyl (blue, red) species. Relative to the former, the latter compounds have a red-shifted peak in the $1300 \mathrm{~cm}^{-1}-1400 \mathrm{~cm}^{-1}$ 
range (naphthalene $\mathrm{C}=\mathrm{C}$ stretching). Several other small differences, specific to 1'-naphthyl and 2'-naphthyl species, are present across the entire fingerprint. Though such distinctions are not easy to make among the N-1,1-dimethylpropyl (black, red) and N-pentyl species (grey, blue), each compound nevertheless has a unique experimental SERS fingerprint. As before, the ability to discriminate among such isomers is unique to Raman/SERS. This adds to its value as a compliment to MS on an integrated, field-based sensing platform. 
(a)
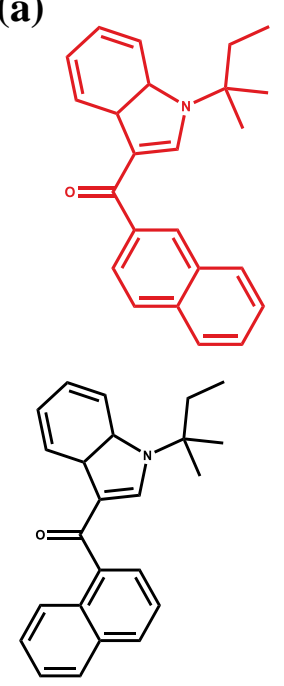

(b)

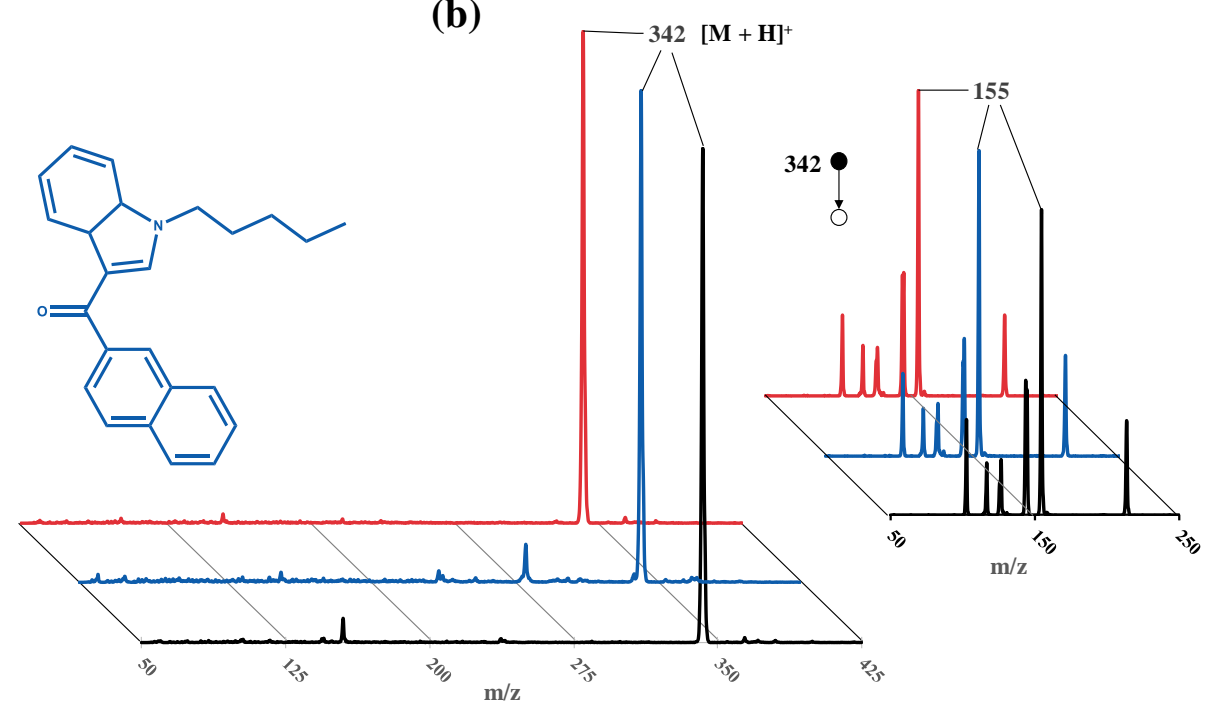

(c)

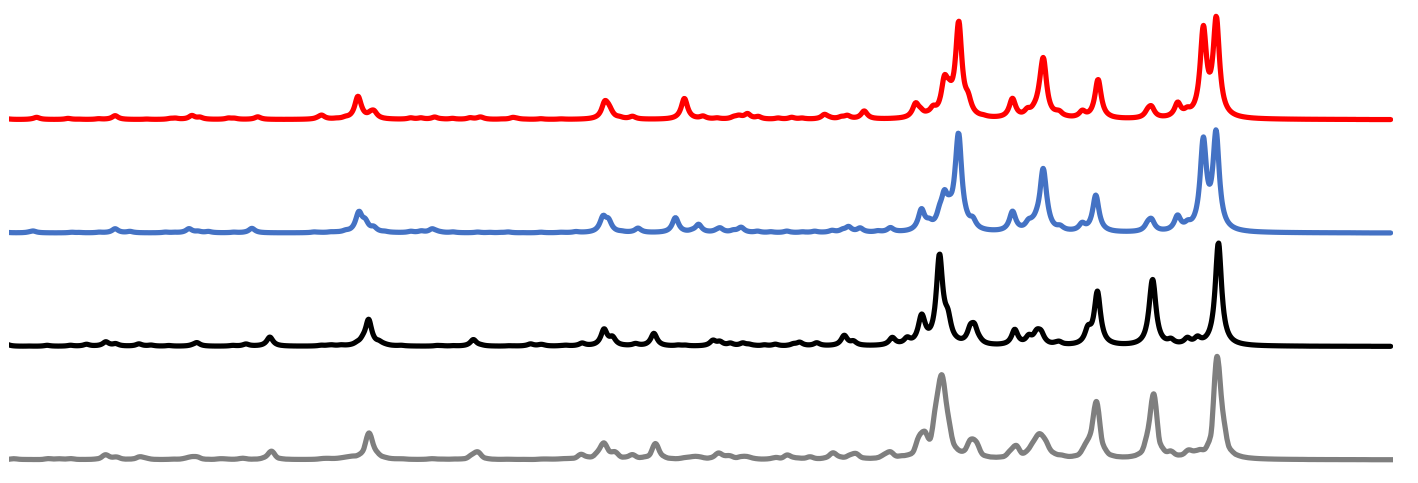

(d)

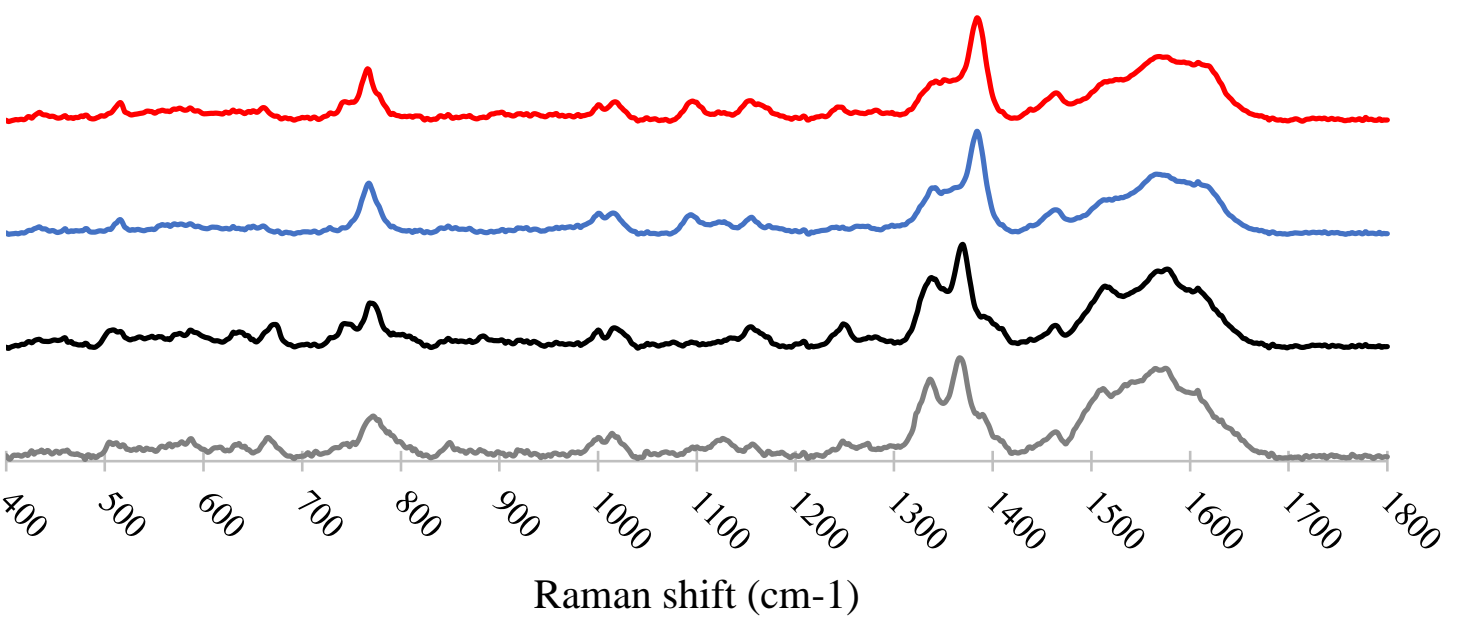

Figure 23. Isomers of JWH-018 (a): N-1,1-dimethylpropyl isomer (black), 2'-naphthyl isomer (blue), and 2'-naphthyl-N-1,1-dimethylpropyl isomer (red). MS and tandem MS spectra (b) are indistinguishable, while simulated (c) and experimental (d) Raman/SERS spectra show similar 
- but still distinct - fingerprints for each of the four compounds. DFT and SERS of underivatized JWH-018 (grey) is also shown.

\section{SERS-PSI-MS}

Having established the potential of SERS detection on paper substrates, the method was finally coupled with PSI-MS. At the outset, dual-method detection had to be optimized. Among the weightier aspects of this process was the decision to collect SERS spectra before, during, or after PSI-MS. Coupled analysis, where SERS data was collected at all three stages, was performed for all five representative compounds.

Figure 24 reveals a characteristic outcome of coupled analysis, with SERS spectra collected before, during, and after PSI-MS. Since the analyte - in this case $100 \mathrm{ng}$ of 2C-B - was dispersed through the paper, the SERS spectrum collected before PSI-MS analysis (Figure 24a) had strong background features. Still, 2C-B was identifiable based on the presence of some of its characteristic peaks $\left(474 \mathrm{~cm}^{-1}, 1290 \mathrm{~cm}^{-1}\right)$. SERS intensity dropped off considerably during PSIMS analysis (Figure 24b) - which is justifiable on the basis of molecules having desorbed from the plasmonic surfaces in the presence of spray solvent. SERS analysis conducted after PSI-MS analysis (Figure 24c) brought forth an unexpected result - a 2C-B fingerprint of excellent quality, with little to no persistence of background peaks (such as the shoulder peak at $1500 \mathrm{~cm}^{-1}$ and the isolated, broad peak at $1160 \mathrm{~cm}^{-1}$, seen in Figure 24a). PSI-MS analysis identified 2C-B based on its protonated molecule and characteristic fragments (Figure 24d, e).

The drastic improvement in the analyte's SERS fingerprint following PSI-MS suggests that analyte adsorption onto the plasmonic surfaces was facilitated by the PSI-MS process. A possible explanation is that the AuNPs are cleaned of pre-adsorbed molecules, while analyte is 
preconcentrated at the paper's tip. This preconcentration - resulting from bulk movement of analyte as solvent wicks through the paper - is consistent with published findings for both PSIMS and SERS. ${ }^{68,95}$ Though current access to instrumentation did not allow for a rigorous investigation of this phenomenon, mapping of the plasmonic papers with a Raman microscope could probe the bulk movement of analyte, possibly allowing optimization of the process as a function of the volume of spray solvent and electrospray duration.

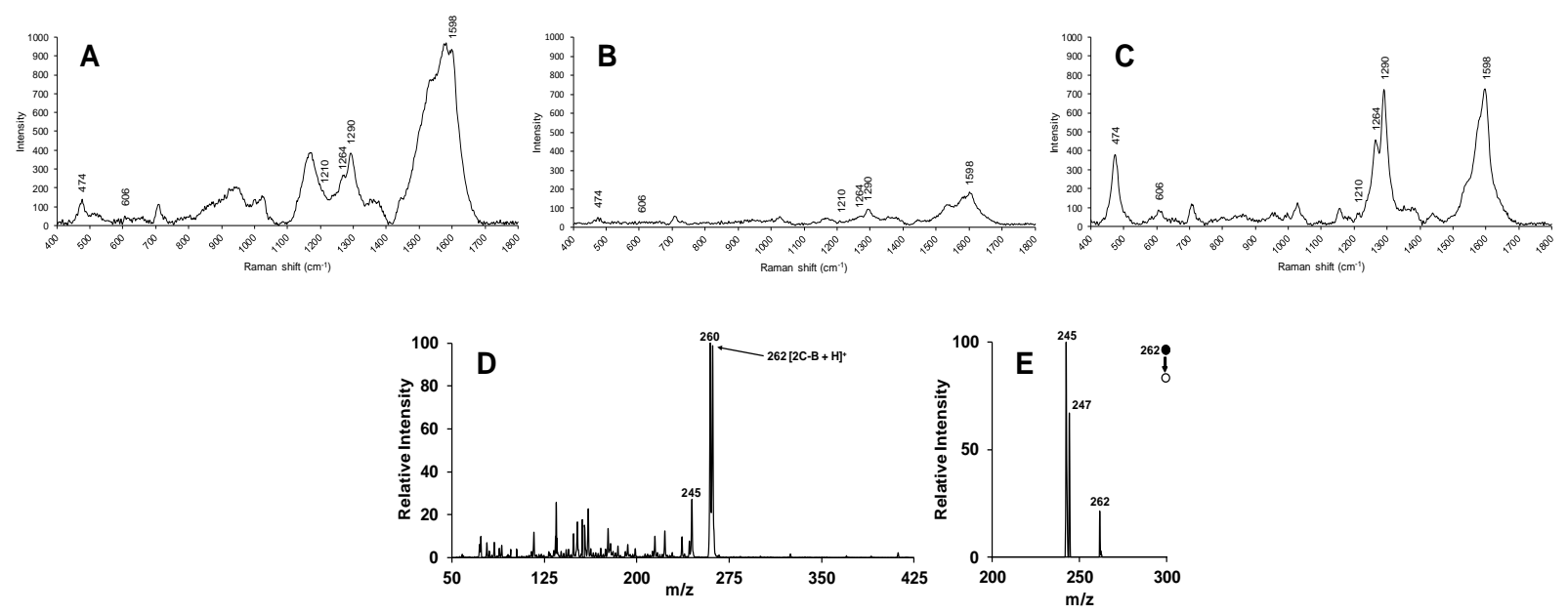

Figure 24. Representative data for SERS-PSI-MS, collected from $50 \mathrm{ng}$ of $2 \mathrm{C}-\mathrm{B}$ on plasmonic paper. SERS collected before (a) and during PSI-MS (b) are of inferior quality to that collected after (c), where characteristic drug peaks are sharper and undesirable background peaks are absent. MS and tandem MS spectra (d, e) are satisfactory for identification purposes, showing the protonated molecule and characteristic fragments, respectively.

Pre-PSI versus post-PSI SERS analysis was performed for each of the five analytes (Figure 25). Similarly to before, all post-PSI-MS SERS spectra were of very high quality. Furthermore, data collected during this phase of experiments suggested that the LODs for SERS could be lowered through performing coupled analysis with SERS collected after PSI-MS. As an 
example, Figure 25c shows the spectrum of $15 \mathrm{ng}$ fentanyl on PSI-cut paper, which, at $\sim 0.4$ $\mathrm{ng} / \mathrm{mm}^{2}$, is very close to its SERS detection limit of $0.14 \mathrm{ng} / \mathrm{mm}^{2}$. SERS collected pre-PSI-MS shows no fingerprint for fentanyl, but collection post-PSI-MS yields a striking fentanyl spectrum. It is also notable that, in the case of hydrocodone (Figure 25d), the absolute intensity of the SERS spectrum collected post-PSI-MS was lower than that collected before. Even in this case, however, the analyte fingerprint was much clearer and easier to identify post-PSI-MS. 

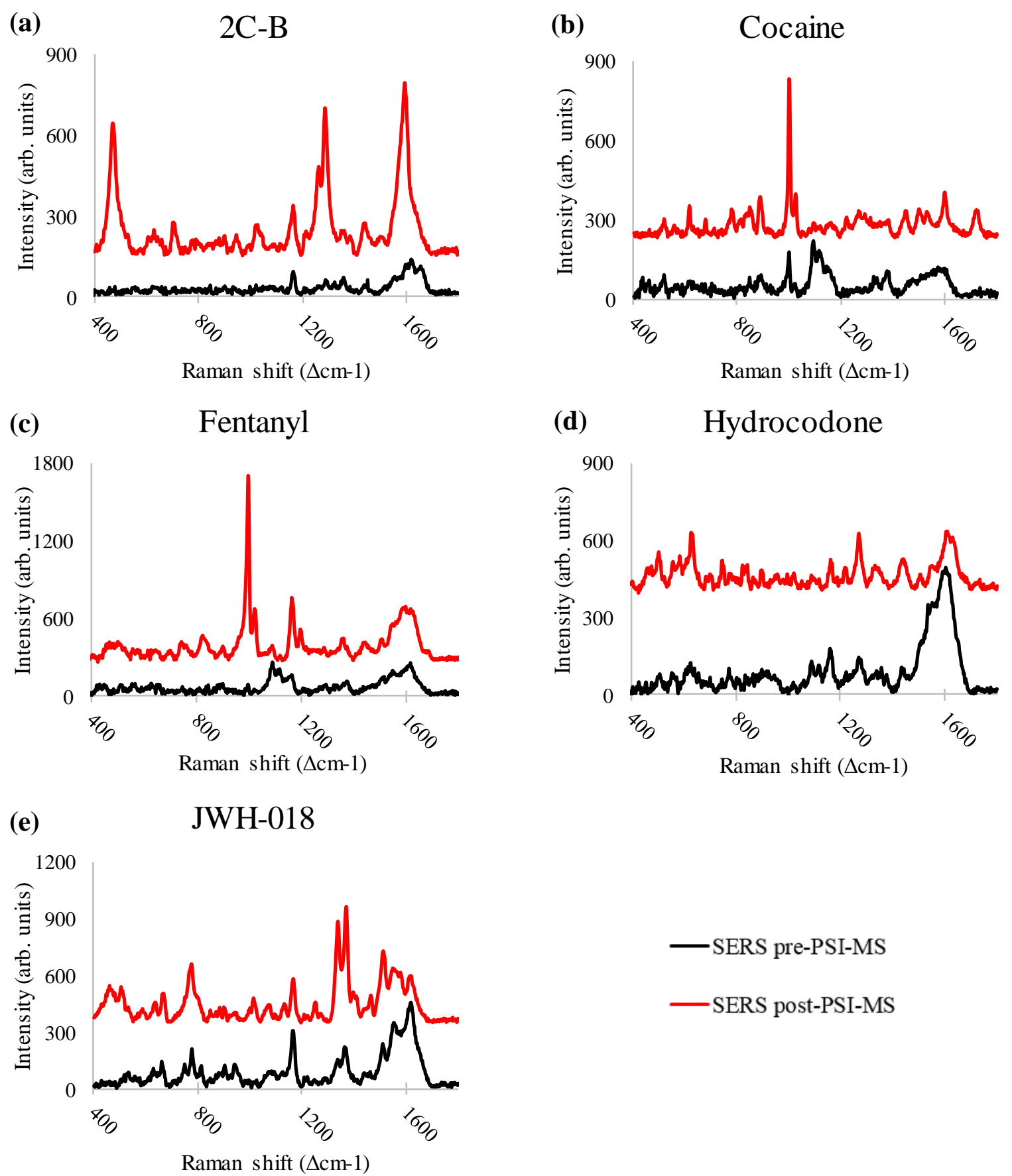

— SERS pre-PSI-MS

- SERS post-PSI-MS

Figure 25. Plasmonic, PSI-cut paper with SERS analysis conducted before and after PSI-MS for all five drugs. Pre-PSI-MS SERS analysis (black traces) was consistently inferior to postPSI-MS SERS analysis (red traces) for identification purposes. Drug was deposited as $50 \mathrm{ng}$ in the case of 2C-B (a), cocaine (b), hydrocodone (d), and JWH-018 (e), while - in order to probe 
the potential for lowering LODs - only $15 \mathrm{ng}$ was deposited for fentanyl (c). It is notable that the absolute intensity of SERS spectra could decrease post-PSI, as in the case of hydrocodone. Still, the analyte fingerprint was easier to identify post-PSI in every case.

Having determined that SERS spectra were far more useful when collected post-PSI, the staging for integrated analysis was constructed to reflect this. A vertical and translational micrometer had already been acquired and integrated into prototypical stage designs. These were then supplemented with 3D-printed components to allow rapid transitioning from PSI-MS to SERS analysis.

Briefly, the updated stage contained a central channel, wherein paper samples, held in printed, polypropylene strips, could slide back and forth, from the mass spectrometer inlet (Figure 6, left) to the focal point of the Raman laser (Figure 6, right). Since the Raman laser was firmly fastened above the stage, there was no need for focusing prior to acquisitions. The two micrometers could be used to adjust the position of the entire stage, in order to obtain a stable electrospray for each sample. Strips could easily be removed and replaced with new plasmonic paper samples. These updates substantially improved throughput, from $\sim 15 \mathrm{~min} / \mathrm{sample}$ on the former prototype to $\sim 2 \mathrm{~min} / \mathrm{sample}$.

\section{Error-rate Study}

Twenty-five sets of 20 samples $(N=500)$ were prepared and analyzed blind. SERS spectra were interpreted as positive for drug based on the visual discernment of a drug analyte's fingerprint, while the lack of any such fingerprint resulted in a negative interpretation. Specifically, each drug was identified by the presence and consistent relative intensities of the SERS peaks listed in Table 5 for each drug. 
Of the 500 samples, only four required a retest due to conflicting SERS and PSI-MS results. Of these, three were successfully confirmed by retesting, while one returned further ambiguity. For the combined technique, this corresponds to a false negative rate $0.2 \%$.

Performing only one test of each sample returned a success rate of $99.2 \%$ overall, and there were no false-positives.

To assess consistency of SERS performance across the study, a ratio between the two most prominent analyte-specific peaks was taken to represent the overall spectral fingerprint for each drug molecule. The average and standard deviation of this ratio were calculated for each drug, on each day. The intra-day variability was evaluated as the relative percent standard deviation $(\% \mathrm{RSD})$ of the ratio within a given day. For example, the pertinent peaks for hydrocodone $-1272 \mathrm{~cm}^{-1}$ and $1436 \mathrm{~cm}^{-1}$ - had an intra-day variability ranging from $1.2 \%-27 \%$. The inter-day variability was calculated as the \%RSD of the average SERS intensity collected from each day. Table 5 summarizes the SERS intra- and inter-day variabilities for each drug.

Table 5

Intra-day and inter-day variabilities in SERS drug identification performance for coupled SERSPSI-MS analysis

\begin{tabular}{ccccc} 
Drug & $\begin{array}{c}\text { SERS peak ratio } \\
\left(\mathbf{c m}^{\left.-1 / \mathbf{c m}^{-1}\right)}\right.\end{array}$ & $\begin{array}{c}\text { Range of intra-day } \\
\text { variability }\end{array}$ & $\begin{array}{c}\text { Average intra-day } \\
\text { variability }\end{array}$ & $\begin{array}{c}\text { Inter-day } \\
\text { variability }\end{array}$ \\
\hline 2C-B & $1290 / 474$ & $0.29-41$ & 15 & 15 \\
Cocaine & $998 / 886$ & $0.13-32$ & 12 & 15 \\
Fentanyl & $998 / 742$ & $0.10-25$ & 13 & 18 \\
Hydrocodone & $1272 / 1436$ & $1.2-27$ & 11 & 15 \\
JWH-018 & $1336 / 772$ & $0.39-11$ & 6.2 & 7.9 \\
\hline
\end{tabular}




\section{CHAPTER IV: CONCLUSIONS AND OUTLOOK}

SERS sensing on plasmonic paper substrates has herein been successfully piloted as a compliment to PSI-MS for the analysis of trace drug residues. Proof-of-principle investigations demonstrated SERS detection for five controlled substances, chosen from among common and emerging drug classes. All the SERS fingerprints showed good conformity to DFT-calculated Raman spectra. Calibration models were constructed for each drug, which placed the LODs primarily in the single-nanogram range - comparable sensitivity to portable PSI-MS for the same analytes. Excellent selectivity - surpassing PSI-MS - was demonstrated in the analysis of isomeric compounds. Different parameters for integrated SERS-PSI-MS analysis were compared, and a standard operating procedure was developed. This procedure was guided by the noteworthy finding that SERS spectral quality was significantly improved when this technique was performed after the PSI-MS process. High-throughput integrated analysis was achieved using a custom-built stage with 3D-printed supports and sample strips. Finally, an error rate study of 500 samples, each containing sub-microgram quantities of drug, produced a $99.8 \%$ success rate.

Several findings in this research merit further study. Among these, the preparation of plasmonic substrates deserves attention. While background signals tended towards a consistent fingerprint, there were frequent, seemingly batch-specific deviations. Of these, some background signals were so strong as to severely obscure the presence of a drug's spectral fingerprint. Since the preparation of papers took a full 24 hours, time limitations (e.g., during the error rate study) did not allow the rejection of batches by way of quality control.

Beyond optimizing the current process of soaking papers in citrate-reduced AuNPs, there are ample alternate methods for preparing plasmonic papers. ${ }^{63}$ Several of these preparations 
involve no added constituents relative to the current process (requiring only $\mathrm{Au}$ or $\mathrm{Ag}$ salts and reducing agent). ${ }^{78,80,96}$ Thus, such methods of preparation could feasibly be explored as compatible with PSI-MS (by not introducing interfering substances), while perhaps improving SERS performance (by way of higher enhancements, lower \%RSDs, and better long-term stability). By far the most helpful substrate would be one with consistent, predictable background signals, as this would be a limiting factor in the success of building and implementing a librarymatching system.

Another worthy point of future study is the translation of plasmonic paper into a fieldable sampling format. The current 3D-printed sample holders were designed to loosely resemble swabs. This theme can be developed towards the realization of a useable 3D-printed, plasmonic paper swab. An interesting, and underexplored, aspect of the current project was the use of nanofibrous polymer membranes for SERS-PSI-MS. There is precedent for improving SERS and PSI-MS separately using PLA (polylactic acid) nanofibrous membranes. ${ }^{97,98}$ Furthermore, PLA is an affordable and widely available 3D-printing material. A combination of PLA nanofibrous membranes and 3D-printed PLA swabs could be investigated as a highly versatile, field-ready SERS-PSI-MS substrate.

Besides developments to the plasmonic substrates, the most immediate future work is the incorporation of multivariate statistical tools to fully utilize SERS spectral data. Such tools are very common in Raman and SERS literature, and enable generation of library-matching systems and analysis of mixtures. For the latter, SERS enjoys no such selectivity as PSI-MS, which with tandem MS capabilities can effectively eliminate all but the desired ions. Rather, SERS spectra must be statistically deconstructed into the summed fingerprints of all the molecular species 
present. Multivariate statistical means of handling such data are needed to usefully situate SERS alongside PSI-MS in the analysis of real-world drug samples.

Overall, SERS-PSI-MS has been demonstrated as a promising tool for modernizing portable drug detection. With pursuit of the aforementioned points, particularly the multivariate utilization of the SERS spectral data, the benefits of this dual-instrument platform could be maximized, bringing it closer to the field and to real-world samples. 


\section{REFERENCES}

(1) Durose, M. R.; United States Bureau of Justice, S. Census of publicly funded forensic crime laboratories, 2005; U.S. Dept. of Justice, Office of Justice Programs: Washington, DC, 2008.

(2) National Research Council Committee on Identifying the Needs of the Forensic Science, C.; National Research Council . Committee on Science, T.; National Research Council Committee on, A.; Theoretical, S. Strengthening forensic science in the United States: a path forward; National Academies Press: Washington, D.C., 2009.

(3) Tsumura, Y.; Mitome, T.; Kimoto, S. Forensic science international 2005, 155, 158.

(4) Namera, A.; Nakamoto, A.; Saito, T.; Nagao, M. Forensic Toxicology 2011, 29, 1.

(5) Armenian, P.; Vo, K. T.; Barr-Walker, J.; Lynch, K. L. Neuropharmacology 2018, 134, 121.

(6) Kloosterman, A.; Mapes, A.; Geradts, Z.; van Eijk, E.; Koper, C.; van den Berg, J.;

Verheij, S.; van der Steen, M.; van Asten, A. Philosophical Transactions of the Royal Society B: Biological Sciences 2015, 370, 20140264.

(7) SWGDRUG 2010.

(8) Krauss, S. T.; Remcho, T. P.; Lipes, S. M.; Aranda, R.; Maynard, H. P.; Shukla, N.; Li, J.; Tontarski, R. E.; Landers, J. P. Analytical Chemistry 2016, 88, 8689.

(9) Musile, G.; Wang, L.; Bottoms, J.; Tagliaro, F.; McCord, B. Analytical methods 2015, 7, 8025.

(10) He, M.; Li, Z.; Ge, Y.; Liu, Z. Analytical Chemistry 2016, 88, 1530.

(11) Walczak, R.; Dziuban, J.; Szczepańska, P.; Scholles, M.; Doyle, H.; Krüger, J.; RuanoLopez, J. Procedia Chemistry 2009, 1, 999. 
(12) de Araujo, W. R.; Cardoso, T. M.; da Rocha, R. G.; Santana, M. H.; Munoz, R. A.;

Richter, E. M.; Paixao, T. R.; Coltro, W. K. Analytica Chimica Acta 2018, 1034, 1.

(13) de Jong, M.; Florea, A.; Vries, A.-M. d.; van Nuijs, A. L. N.; Covaci, A.; Van Durme, F.;

Martins, J. C.; Samyn, N.; De Wael, K. Analytical Chemistry 2018, 90, 5290.

(14) Andrade, A. F. B.; Mamo, S. K.; Gonzalez-Rodriguez, J. Analytical Chemistry 2017, 89, 1445.

(15) Dronova, M.; Smolianitski, E.; Lev, O. Analytical Chemistry 2016, 88, 4487.

(16) Wang, J.; Taha, Z. H.; Google Patents: 1993.

(17) Richter, E. M.; Tormin, T. F.; Cunha, R. R.; Silva, W. P.; Pérez-Junquera, A.; FanjulBolado, P.; Hernández-Santos, D.; Muñoz., R. A. A. Electroanalysis 2016, 28, 1856.

(18) Asturias-Arribas, L.; Alonso-Lomillo, M. A.; Domínguez-Renedo, O.; Arcos-Martínez, M. J. Talanta 2013, 105, 131.

(19) Vidal, J. C.; Bertolín, J. R.; Bonel, L.; Asturias, L.; Arcos-Martínez, M. J.; Castillo, J. R. Electroanalysis 2016, 28, 685.

(20) Abnous, K.; Danesh, N. M.; Ramezani, M.; Taghdisi, S. M.; Emrani, A. S. Sensors and Actuators B: Chemical 2016, 224, 351.

(21) Salles, M. O.; Araujo, W. R.; Paixão, T. R. Journal of the Brazilian Chemical Society 2016, 27, 54 .

(22) Balbino, M. A.; Oiye, É. N.; Ribeiro, M. F. M.; Júnior, J. W. C.; Eleotério, I. C.; Ipólito, A. J.; de Oliveira, M. F. Journal of Solid State Electrochemistry 2016, 20, 2435.

(23) Souza, G. A.; Arantes, L. C.; Guedes, T. J.; de Oliveira, A. C.; Marinho, P. A.; Muñoz, R. A. A.; dos Santos, W. T. P. Electrochemistry Communications 2017, 82, 121. 
(24) Cooks, R. G.; Ouyang, Z.; Takats, Z.; Wiseman, J. M. Science (New York, N.Y.) 2006, 311, 1566.

(25) Ma, X.; Ouyang, Z. Trends in analytical chemistry : TRAC 2016, 85, 10.

(26) Monge, M. E.; Harris, G. A.; Dwivedi, P.; Fernandez, F. M. Chemical reviews 2013, 113, 2269.

(27) Snyder, D. T.; Pulliam, C. J.; Ouyang, Z.; Cooks, R. G. Analytical Chemistry 2016, 88, 2.

(28) Morelato, M.; Beavis, A.; Kirkbride, P.; Roux, C. Forensic science international 2013, $226,10$.

(29) Takats, Z.; Wiseman, J. M.; Gologan, B.; Cooks, R. G. Science (New York, N.Y.) 2004, $306,471$.

(30) Brown, H.; Oktem, B.; Windom, A.; Doroshenko, V.; Evans-Nguyen, K. Forensic Chemistry 2016, 1, 66.

(31) Cody, R. B.; Laramee, J. A.; Durst, H. D. Analytical Chemistry 2005, 77, 2297.

(32) Harper, J. D.; Charipar, N. A.; Mulligan, C. C.; Zhang, X.; Cooks, R. G.; Ouyang, Z. Analytical Chemistry 2008, 80, 9097.

(33) Martinez-Jarquin, S.; Winkler, R. TrAC Trends in Analytical Chemistry 2017, 89, 133.

(34) Wang, H.; Liu, J.; Cooks, R. G.; Ouyang, Z. Angewandte Chemie International Edition 2010, 49, 877.

(35) Silva, L. C. d.; Pereira, I.; Carvalho, T. C. d.; Allochio Filho, J. F.; Romão, W.; Gontijo Vaz, B. Analytical methods 2019, 11, 999.

(36) Zhang, Z.; Cooks, R. G.; Ouyang, Z. Analyst 2012, 137, 2556. 
(37) Lawton, Z. E.; Traub, A.; Fatigante, W. L.; Mancias, J.; O’Leary, A. E.; Hall, S. E.;

Wieland, J. R.; Oberacher, H.; Gizzi, M. C.; Mulligan, C. C. Journal of the American Society for Mass Spectrometry 2017, 28, 1048.

(38) Mulligan, C. C.; O'Leary, A. E. Accessing the Probative Value of Physical Evidence at Crime Scenes with Ambient Mass Spectrometry and Portable Instrumentation; US Department of Justice, Office of Justice Programs, National Institute of .., 2015.

(39) O'Leary, A. E.; Hall, S. E.; Vircks, K. E.; Mulligan, C. C. Analytical methods 2015, 7, 7156.

(40) O'Leary, A. E.; Oberacher, H.; Hall, S. E.; Mulligan, C. C. Analytical methods 2015, 7, 3331.

(41) Mulligan, C. C.; Wieland, J. R.; Gizzi, M. C. Analytical Validation and Impact Assessment of On-site Evidence Screening Via Ambient Sampling, Portable Mass Spectrometry: Award No. 2015-IJ-CX-K011: Final Summary Overview; Illinois State University, 2018.

(42) Warner, R. M. Applied statistics: From bivariate through multivariate techniques; Sage, 2008.

(43) Christie, R.; Horan, E.; Fox, J.; O'Donnell, C.; Byrne, H.; McDermott, S.; Power, J.; Kavanagh, P. Drug testing and analysis 2014, 6, 651.

(44) Guirguis, A.; Girotto, S.; Berti, B.; Stair, J. L. Forensic science international 2017, 273, 113.

(45) Chalmers, J. M.; Edwards, H. G.; Hargreaves, M. D. Infrared and Raman spectroscopy in forensic science; John Wiley \& Sons, 2012.

(46) Choo-Smith, L.-P.; Maquelin, K.; Van Vreeswijk, T.; Bruining, H.; Puppels, G.; Thi, N. N.; Kirschner, C.; Naumann, D.; Ami, D.; Villa, A. Appl. Environ. Microbiol. 2001, 67, 1461. 
(47) Huang, W. E.; Griffiths, R. I.; Thompson, I. P.; Bailey, M. J.; Whiteley, A. S. Analytical Chemistry 2004, 76, 4452.

(48) Kalasinsky, K. S.; Hadfield, T.; Shea, A. A.; Kalasinsky, V. F.; Nelson, M. P.; Neiss, J.; Drauch, A. J.; Vanni, G. S.; Treado, P. J. Analytical Chemistry 2007, 79, 2658.

(49) Maquelin, K.; Choo-Smith, L. P.; van Vreeswijk, T.; Endtz, H. P.; Smith, B.; Bennett, R.; Bruining, H. A.; Puppels, G. J. Analytical Chemistry 2000, 72, 12.

(50) Maquelin, K.; Choo-Smith, L.-P.; Endtz, H. P.; Bruining, H.; Puppels, G. Journal of Clinical Microbiology 2002, 40, 594.

(51) Hajjou, M.; Qin, Y.; Bradby, S.; Bempong, D.; Lukulay, P. Journal of pharmaceutical and biomedical analysis 2013, 74, 47.

(52) Paudel, A.; Raijada, D.; Rantanen, J. Advanced drug delivery reviews 2015, 89, 3.

(53) Sorak, D.; Herberholz, L.; Iwascek, S.; Altinpinar, S.; Pfeifer, F.; Siesler, H. W. Applied Spectroscopy Reviews 2012, 47, 83.

(54) Hargreaves, M. D.; Page, K.; Munshi, T.; Tomsett, R.; Lynch, G.; Edwards, H. G. Journal of Raman Spectroscopy: An International Journal for Original Work in all Aspects of Raman Spectroscopy, Including Higher Order Processes, and also Brillouin and Rayleigh Scattering 2008, 39, 873.

(55) Izake, E. L. Forensic science international 2010, 202, 1.

(56) de Oliveira Penido, C. A. F.; Pacheco, M. T. T.; Lednev, I. K.; Silveira Jr, L. Journal of Raman Spectroscopy 2016, 47, 28.

(57) Albrecht, M. G.; Creighton, J. A. Journal of the american chemical society 1977, 99, 5215. 
(58) Jeanmaire, D. L.; Van Duyne, R. P. Journal of electroanalytical chemistry and interfacial electrochemistry 1977, 84, 1.

(59) Kneipp, K.; Wang, Y.; Kneipp, H.; Perelman, L. T.; Itzkan, I.; Dasari, R. R.; Feld, M. S. Physical review letters 1997, 78, 1667.

(60) Nie, S.; Emory, S. R. science 1997, 275, 1102.

(61) Le Ru, E.; Blackie, E.; Meyer, M.; Etchegoin, P. G. The Journal of Physical Chemistry C 2007, 111, 13794.

(62) Bell, S. E.; Sirimuthu, N. M. Chemical Society Reviews 2008, 37, 1012.

(63) Prikhozhdenko, E. S.; Bratashov, D. N.; Gorin, D. A.; Yashchenok, A. M. Nano Research 2018, 11, 4468.

(64) Fan, M.; Andrade, G. F. S.; Brolo, A. G. Analytica Chimica Acta 2011, 693, 7.

(65) Lin, X.-M.; Cui, Y.; Xu, Y.-H.; Ren, B.; Tian, Z.-Q. Analytical and Bioanalytical Chemistry 2009, 394, 1729.

(66) Norrod, K. L.; Sudnik, L. M.; Rousell, D.; Rowlen, K. L. Applied Spectroscopy 1997, 51, 994.

(67) Restaino, S. M.; White, I. M. Analytica Chimica Acta 2019, 1060, 17.

(68) Hoppmann, E. P.; Yu, W. W.; White, I. M. Methods 2013, 63, 219.

(69) Webb, J. A.; Aufrecht, J.; Hungerford, C.; Bardhan, R. Journal of Materials Chemistry C 2014, 2, 10446.

(70) Yu, W. W.; White, I. M. The Analyst 2013, 138, 3679.

(71) Haddad, A.; Comanescu, M. A.; Green, O.; Kubic, T. A.; Lombardi, J. R. Analytical Chemistry 2018, 90, 12678.

(72) Ngo, Y. H.; Li, D.; Simon, G. P.; Garnier, G. Langmuir 2012, 28, 8782. 
(73) Li, B.; Zhang, W.; Chen, L.; Lin, B. ELECTROPHORESIS 2013, 34, 2162.

(74) Yu, W. W.; White, I. M. Analytical Chemistry 2010, 82, 9626.

(75) Lin, S.; Hasi, W.-L.-J.; Lin, X.; Han, S.-q.-g.-w.; Lou, X.-T.; Yang, F.; Lin, D.-Y.; Lu, Z.-W. Analytical methods 2015, 7, 5289.

(76) Cheng, M.-L.; Tsai, B.-C.; Yang, J. Analytica Chimica Acta 2011, 708, 89.

(77) Rajapandiyan, P.; Yang, J. Journal of Raman Spectroscopy 2014, 45, 574.

(78) He, S.; Chua, J.; Tan, E. K. M.; Kah, J. C. Y. RSC Advances 2017, 7, 16264.

(79) Lee, C. H.; Tian, L.; Singamaneni, S. ACS Applied Materials \& Interfaces 2010, 2, 3429.

(80) Kim, W.; Lee, J.-C.; Shin, J.-H.; Jin, K.-H.; Park, H.-K.; Choi, S. Analytical Chemistry 2016, 88,5531 .

(81) Fikiet, M. A.; Khandasammy, S. R.; Mistek, E.; Ahmed, Y.; Halámková, L.; Bueno, J.; Lednev, I. K. Spectrochimica Acta Part A: Molecular and Biomolecular Spectroscopy 2018, $197,255$.

(82) Muehlethaler, C.; Leona, M.; Lombardi, J. R. Analytical Chemistry 2016, 88, 152.

(83) Mostowtt, T.; McCord, B. Talanta 2017, 164, 396.

(84) Kline, N. D.; Tripathi, A.; Mirsafavi, R.; Pardoe, I.; Moskovits, M.; Meinhart, C.;

Guicheteau, J. A.; Christesen, S. D.; Fountain, A. W. Analytical Chemistry 2016, 88, 10513.

(85) Rana, V.; Cañamares, M. V.; Kubic, T.; Leona, M.; Lombardi, J. R. Journal of forensic sciences 2011, 56, 200.

(86) Trachta, G.; Schwarze, B.; Brehm, G.; Schneider, S.; Hennemann, M.; Clark, T. Journal of Raman Spectroscopy 2004, 35, 368.

(87) Dana, K.; Shende, C.; Huang, H.; Farquharson, S. Journal of analytical \& bioanalytical techniques 2015, 6, 1. 
(88) Inscore, F.; Shende, C.; Sengupta, A.; Huang, H.; Farquharson, S. Applied Spectroscopy 2011, 65, 1004.

(89) Nuntawong, N.; Eiamchai, P.; Somrang, W.; Denchitcharoen, S.; Limwichean, S.;

Horprathum, M.; Patthanasettakul, V.; Chaiya, S.; Leelapojanaporn, A.; Saiseng, S. Sensors and Actuators B: Chemical 2017, 239, 139.

(90) Andersson, M. P.; Uvdal, P. The Journal of Physical Chemistry A 2005, 109, 2937.

(91) Huang, Z.; Nagpal, A.; Siddhanta, S.; Barman, I. Journal of Raman Spectroscopy 2018, $49,1552$.

(92) Goodacre, R.; Graham, D.; Faulds, K. Trends in Analytical Chemistry 2018, 102, 359.

(93) Scott, A. P.; Radom, L. The Journal of Physical Chemistry 1996, 100, 16502.

(94) Zvereva, E. E.; Shagidullin, A. R.; Katsyuba, S. A. The Journal of Physical Chemistry A 2011, 115, 63.

(95) Yang, Q.; Wang, H.; Maas, J. D.; Chappell, W. J.; Manicke, N. E.; Cooks, R. G.; Ouyang, Z. International Journal of Mass Spectrometry 2012, 312, 201.

(96) Wang, J.; Yang, L.; Liu, B.; Jiang, H.; Liu, R.; Yang, J.; Han, G.; Mei, Q.; Zhang, Z. Analytical Chemistry 2014, 86, 3338.

(97) Lai, P.-H.; Chen, P.-C.; Liao, Y.-W.; Liu, J.-T.; Chen, C.-C.; Lin, C.-H. International Journal of Mass Spectrometry 2015, 375, 14.

(98) Szymborski, T.; Witkowska, E.; Adamkiewicz, W.; Waluk, J.; Kamińska, A. Analyst 2014, 139, 5061. 